\title{
On The Eigenfilter Design Method and Its Applications: A Tutorial
}

\author{
Andre Tkacenko, Student Member, IEEE, P. P. Vaidyanathan, Fellow, IEEE, and \\ Truong Q. Nguyen, Senior Member, IEEE
}

\begin{abstract}
The eigenfilter method for digital filter design involves the computation of filter coefficients as the eigenvector of an appropriate Hermitian matrix. Because of its low complexity as compared to other methods as well as its ability to incorporate various time and frequency-domain constraints easily, the eigenfilter method has been found to be very useful. In this paper, we present a review of the eigenfilter design method for a wide variety of filters, including linear-phase finite impulse response (FIR) filters, nonlinear-phase FIR filters, all-pass infinite impulse response (IIR) filters, arbitrary response IIR filters, and multidimensional filters. Also, we focus on applications of the eigenfilter method in multistage filter design, spectral/spacial beamforming, and in the design of channel-shortening equalizers for communications applications.
\end{abstract}

Index Terms -Channel-shortening equalizers, constrained filter design, eigenfilter, least-squares filter design.

\section{INTRODUCTION}

$\mathbf{T}$ HE EIGENFILTER design method for discrete time filters involves the determination of filter coefficients as the eigenvector of a particular Hermitian positive definite (and often real and symmetric) matrix. As opposed to other filter design algorithms such as the least-squares approach [48], which requires the computation of a matrix inverse, the eigenfilter method only requires the computation of a single eigenvector, which can be found efficiently via the iterative power method [49]. In addition to its inherently low design complexity, the eigenfilter method can also incorporate a variety of time and frequency-domain constraints into the design problem with relative ease, in contrast to other well known filter design methods such as the McClellan-Parks algorithm [16]. Furthermore, because of the myriad of design problems that can be posed as an eigenfilter problem, the method has been shown to be useful for a variety of applications, ranging from spectral/spacial filtering or beamforming to communications regarding the design of channel-shortening equalizers for discrete multitone (DMT) systems.

The notion of such a filter design technique was introduced by Slepian in 1978 [42] in the context of the design of window functions for the ideal low-pass filter response. Slepian considered the problem of designing a window with a minimum stop-

Manuscript received February 5, 2003; revised April 23, 2003. This work was supported in part by the Office of Naval Research Grant N00014-99-1-1002.

A. Tkacenko and P. P. Vaidyanathan are with the Department of Electrical Engineering, California Institute of Technology, Pasadena, CA 91125 USA (e-mail: andre@systems.caltech.edu).

T. Q. Nguyen is with the Department of Electrical and Computer Engineering, University of California at San Diego, La Jolla, CA 92093 USA.

Digital Object Identifier 10.1109/TCSII.2003.816942 band energy (subject to a unit norm constraint on the window coefficients to avoid a trivial solution). It was found that the optimal window coefficients could be found from the eigenvector of a real, symmetric, positive definite, Toeplitz matrix corresponding to its smallest eigenvalue.

In 1987, Vaidyanathan and Nguyen [50] generalized Slepian's method for window design to the design of linear-phase finite impulse response (FIR) filters and formally introduced the eigenfilter design method. They generalized Slepian's method to account for both passband and stopband conditions and showed how to design a variety of filters (mainly low pass, high pass, and band pass) with various time- and frequency-domain constraints, including the Nyquist constraint and flatness constraints [49]. Numerous simulation results were provided showing the power and usefulness of the eigenfilter method.

Since then, various generalizations of the eigenfilter method have been proposed. The design of linear-phase FIR Hilbert transformers and arbitrary order digital differentiators was considered by Pei and Shyu in [24], [25], [28], [36]. More general FIR design methods were considered by Nguyen as well as Pei and Shyu, including the design of linear-phase filters with arbitrary amplitude response [20], the design of arbitrary complex coefficient nonlinear-phase filters [27], [29], [21], and the design of $M$ th band nonlinear-phase filters by Wisutmethangoon and Nguyen [52]. Multidimensional extensions to the eigenfilter method (mostly two dimensional) were originally proposed by Nashashibi and Charalambous [18] and later considered by Pei and others [26], [30], [31], [35], [8]. The eigenfilter method has even been used to design infinite impulse response (IIR) filters, including all-pass filters [14], [41], [33], [22], [55], arbitrary IIR filters using a time-domain [32] and a frequency-domain approach [1], [2], and IIR filters with time and frequency-domain constraints [39]. Recently, eigenfilter methods for filters with general linear constraints were proposed [37] along with methods based on a total least-squares-error criterion [38], [54].

As many design problems can be posed as an eigenfilter problem, the eigenfilter method has been found to be useful for several applications. For example, it has been used to design multistage interpolated FIR (IFIR) filters [9] as well as arbitrary $\log$ magnitude and phase response filters [34]. In addition, it has been used to develop prototype filters for pseudo-quadrature mirror filter (pseudo-QMF) uniform and nonuniform cosine modulated filter banks [3], [4]. The generalization of the eigenfilter approach to solve general least-squares approximation problems was proposed in [10]. Applications to spectral/spatial filtering were also shown in [10] and more recently in [11]. 
In addition, the eigenfilter method has been found useful for cancelling selective signals in an acoustic environment [6], [7]. Use of this method for image scaling or size conversion was shown in [53]. Recently, the eigenfilter method has been shown to be very important in communications, especially for the design of channel-shortening equalizers in DMT systems [43]. Melsa et al. [17] were the first to apply the eigenfilter method to the problem of channel shortening. Since then, many other eigenfilter based channel-shortening equalizers have been proposed [40], [45]-[47], [5]. Several of these methods have been found to perform nearly optimally in terms of the observed bit rate.

\section{A. Outline}

This paper is organized as follows. In Section II, we give a brief review of the early work on eigenfilters and focus mainly on the work of Slepian [42] and Vaidyanathan and Nguyen [50]. Various generalizations of the eigenfilter approach for filter design are discussed in Section III. In Section IV, applications of the eigenfilter method are considered, such as multistage filter design, spectral/spacial beamforming, and channel shortening for communications. Finally, concluding remarks are made in Section V.

\section{B. Notations}

All notations are as in [49] unless mentioned otherwise. In particular, $(*),(T)$, and $(\dagger)$ correspond, respectively, to the conjugate, transpose, and conjugate transpose operations. Vectors and matrices are in bold font with lowercase letters corresponding to vectors and uppercase letters to matrices. The $k$ th element of a vector $\mathbf{v}$ will be denoted by $[\mathbf{v}]_{k}$, whereas the $(k, l)$ th element of a matrix $\mathbf{P}$ will be denoted by $[\mathbf{P}]_{k, l}$.

\section{HISTORICAL BACKGROUND}

\section{A. Prolate Spheroidal Wave Sequences}

A classical approach for designing FIR low-pass filters is the windowing method [49], in which the impulse response of an ideal low-pass filter is multiplied by a window function $v(n)$. Typically, the window is also low-pass and it turns out that two parameters of the frequency response of the window $V\left(e^{j \omega}\right)$ most prominently affect the quality of the overall response [49]. As the main lobe width of $V\left(e^{j \omega}\right)$ increases, the transition bandwidth of the windowed response likewise tends to get larger, while as the peak sidelobe level of $V\left(e^{j \omega}\right)$ increases, the peak passband and stopband ripples of the overall response also tend to as well. To mitigate the sidelobe level effects of $V\left(e^{j \omega}\right)$, in [42], Slepian considered designing a real window of unit norm for which the energy in the frequency region $\sigma \leq \omega \leq \pi$ is minimized, for some $\sigma$ with $0<\sigma<\pi$. Such a window is a discrete time prolate spheroidal wave function, also called a prolate spheroidal wave sequence [49]. Slepian showed that the coefficients of a prolate spheroidal wave sequence could be obtained from the eigenvector corresponding to the smallest eigenvalue of a real, symmetric, positive definite, Toeplitz matrix, as we now show.

Suppose that $v(n)$ is a real causal sequence of length $N+1$ for some nonnegative integer $N$. Then, the $z$ transform of $v(n)$, namely $V(z)$, is a real coefficient polynomial in $z^{-1}$ of order $N$ given by $V(z)=\sum_{n=0}^{N} v(n) z^{-n}$. The prolate spheroidal wave sequence is the one that minimizes

$$
\phi_{S} \triangleq \int_{\sigma}^{\pi}\left|V\left(e^{j \omega}\right)\right|^{2} \frac{d \omega}{\pi}
$$

subject to the unit norm constraint

$$
\sum_{n=0}^{N} v^{2}(n)=1
$$

If we define the following $(N+1) \times 1$ column vectors:

$$
\begin{aligned}
\mathbf{v} & \triangleq\left[\begin{array}{llll}
v(0) & v(1) & \cdots & v(N)
\end{array}\right]^{T} \\
\mathbf{e}(z) & \triangleq\left[\begin{array}{llll}
1 & z^{-1} & \cdots & z^{-N}
\end{array}\right]^{T}
\end{aligned}
$$

then $V(z)=\mathbf{v}^{T} \mathbf{e}(z)$ and $\left|V\left(e^{j \omega}\right)\right|^{2}=\mathbf{v}^{T} \mathbf{e}\left(e^{j \omega}\right) \mathbf{e}^{\dagger}\left(e^{j \omega}\right) \mathbf{v}$ (as $v(n)$ is real). Using this in (1) yields

$$
\phi_{S}=\mathbf{v}^{T} \mathbf{R v}, \text { where } \mathbf{R}=\int_{\sigma}^{\pi} \mathbf{e}\left(e^{j \omega}\right) \mathbf{e}^{\dagger}\left(e^{j \omega}\right) \frac{d \omega}{\pi} .
$$

As $\mathbf{R}$ is clearly Hermitian, we can decompose $\mathbf{R}$ as $\mathbf{R}=\mathbf{P}+$ $j \mathbf{Q}$, where $\mathbf{P}$ and $\mathbf{Q}$ are, respectively, real symmetric and antisymmetric matrices [12]. Then, as $\mathbf{v}$ is real, we have $\mathbf{v}^{T} \mathbf{Q v}=$ 0 , and so from (3) we get

$$
\phi_{S}=\mathbf{v}^{T} \mathbf{P v} .
$$

The $(k, l)$ th element of $\mathbf{P}$ is given by

$$
\begin{aligned}
{[\mathbf{P}]_{k, l} } & =\int_{\sigma}^{\pi} \cos (k-l) \omega \frac{d \omega}{\pi} \\
& =\delta(k-l)-\frac{\sigma}{\pi} \operatorname{sinc}\left(\frac{\sigma}{\pi}(k-l)\right), 0 \leq k, l \leq N .
\end{aligned}
$$

Clearly, $\mathbf{P}$ is a Toeplitz matrix (as $[\mathbf{P}]_{k, l}$ depends only on $(k-$ $l)$ ), in addition to being real, symmetric, and positive definite (as $\phi_{S}>0$ ) [12]. The unit norm constraint of (2) is equivalent to the statement

$$
\mathbf{v}^{T} \mathbf{v}=1 .
$$

Combining (4) and (6), the optimization problem can be posed as follows:

$$
\text { Minimize } \phi_{S}=\mathbf{v}^{T} \mathbf{P v} \text {, subject to } \mathbf{v}^{T} \mathbf{v}=1 \text {. }
$$

As $\mathbf{P}$ is Hermitian, it follows by Rayleigh's principle [12] that the minimum value of $\phi$ is $\lambda_{0}$ where $\lambda_{0}$ is the smallest eigenvalue of $\mathbf{P}$. Furthermore, this minimum value is achieved if $\mathbf{v}=\mathbf{v}_{0}$, where $\mathbf{v}_{0}$ denotes a unit norm eigenvector of $\mathbf{P}$ corresponding to $\lambda_{0}$. (More generally, $\phi=\lambda_{0}$ iff $\mathbf{v}$ is any unit norm vector in the eigenspace corresponding to $\lambda_{0}$. However, for sake of clarity, we will ignore this scenario.)

The magnitude response of a prolate spheroidal wave sequence obtained using Rayleigh's principle is shown in Fig. 1. Here, we chose the order to be $N=32$ and the frequency parameter as $\sigma=0.2 \pi$. As can be seen from Fig. 1, the window exhibits a large attenuation near $f=0.1$ or $\omega=\sigma=0.2 \pi$ and most of the energy is concentrated in the region $0 \leq \omega \leq \sigma$ as expected. Windows designed using the above method were 


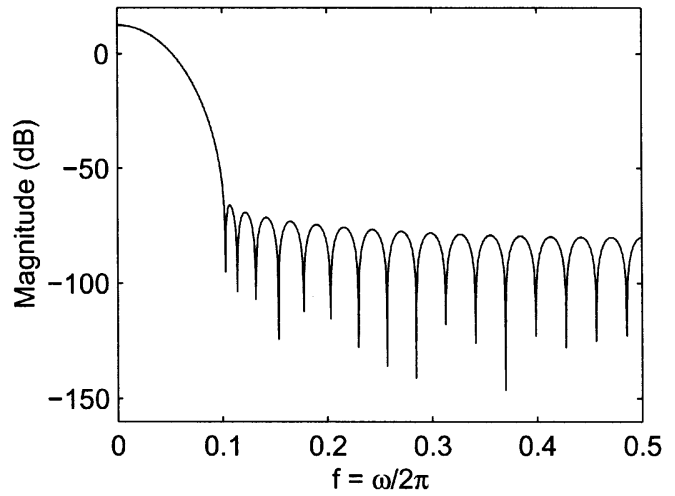

Fig. 1. Magnitude response of a prolate spheroidal wave sequence $(N=32$, $\sigma=0.2 \pi)$.

shown to yield good windowed low-pass FIR filters. Slepian's approach for calculating such sequences paved the way for more general filters to be designed via Rayleigh's principle.

\section{B. Transition From Optimal Windows to Eigenfilters}

Although designing FIR filters using the window method is easy, in general the resulting filters are not optimal in any sense, even if the window is optimal in some sense. Furthermore, we may be interested in filters other than low-pass filters, in which case it is not immediately clear how to use the window method.

1) The Least-Squares Approach for FIR Filter Design: Often, we would like the amplitude response of a real coefficient FIR filter $h(n)$ to approximate a real desired response, say $D(\omega)$, over a particular frequency region $\mathcal{R} \subseteq[0, \pi]$. To prevent phase distortion, we constrain $h(n)$ to have linear phase. For simplicity, we will focus on Type 1 linear-phase filters here, although other types can be used as well. In this case, $h(n)$ is of order $N$, i.e., $H(z)=\sum_{n=0}^{N} h(n) z^{-n}$, where $N$ is even, and $h(n)$ satisfies the symmetry condition $h(n)=h(N-n)$ [49]. As such, $H\left(e^{j \omega}\right)$ is of the form $H\left(e^{j \omega}\right)=e^{-j \omega N / 2} H_{R}(\omega)$, where $H_{R}(\omega)$ is the amplitude response of $H\left(e^{j \omega}\right)$. Here, $H_{R}(\omega)$ is of the form

$$
H_{R}(\omega)=\sum_{n=0}^{M} b_{n} \cos \omega n
$$

where $M=N / 2$ and $b_{n}$ is given by

$$
b_{n}= \begin{cases}2 h(M-n), & 1 \leq n \leq M \\ h(M) & n=0\end{cases}
$$

One approach to get $H_{R}(\omega)$ to approximate the desired response $D(\omega)$ over the region $\mathcal{R}$ is to choose the coefficients $\left\{b_{n}\right\}$ to minimize the mean-squared error between $D(\omega)$ and $H_{R}(\omega)$, given by

$$
\xi_{\mathrm{LS}} \triangleq \frac{1}{\pi} \int_{\mathcal{R}}\left[D(\omega)-H_{R}(\omega)\right]^{2} d \omega .
$$

This method for filter design is known as the least-squares approach [48]. A slight generalization is to incorporate a non- negative weighting function $W(\omega)$ in (9), yielding a weighted mean-squared-error measure

$$
\xi_{\mathrm{WLS}} \triangleq \frac{1}{\pi} \int_{\mathcal{R}} W(\omega)\left[D(\omega)-H_{R}(\omega)\right]^{2} d \omega .
$$

By defining the following $(M+1) \times 1$ vectors:

$$
\begin{aligned}
\mathbf{b} & \triangleq\left[\begin{array}{llll}
b_{0} & b_{1} & \cdots & b_{M}
\end{array}\right]^{T} \\
\mathbf{c}(\omega) & \triangleq\left[\begin{array}{llll}
1 & \cos \omega & \cdots & \cos M \omega
\end{array}\right]^{T}
\end{aligned}
$$

we have $H_{R}(\omega)=\mathbf{b}^{T} \mathbf{c}(\omega)$ from (7), and it can be shown [48] that the optimal coefficient vector $\mathbf{b}$ which minimizes the objective function in (10) is given by

$$
\mathbf{b}=\mathbf{A}^{-1} \mathbf{d}
$$

where $\mathbf{A}$ is an $(M+1) \times(M+1)$ matrix and $\mathbf{d}$ is an $(M+1) \times 1$ vector given by

$$
\begin{aligned}
\mathbf{A} & \triangleq \frac{1}{\pi} \int_{\mathcal{R}} W(\omega) \mathbf{c}(\omega) \mathbf{c}^{T}(\omega) d \omega \\
\mathbf{d} & \triangleq \frac{1}{\pi} \int_{\mathcal{R}} W(\omega) D(\omega) \mathbf{c}(\omega) d \omega .
\end{aligned}
$$

This solution can be obtained by using the trick of completing the square [12].

Though the filter coefficient vector $\mathbf{b}$ from (11) is optimal in the least-squares sense, its computation requires the calculation of a matrix inverse which is high in complexity and subject to numerical inaccuracies. Several approaches have been made to overcome these problems. In [23], Okuda et al. proposed a recursive method based on constructing a set of orthonormal functions. Their method was shown to be both lower in complexity and less susceptible to numerical errors than the conventional least-squares design method in that no direct matrix inversion is involved. Despite these advantages, their method suffers from the drawback that the actual filter coefficients must be found from the coefficients of the orthonormal function expansion, which adds to the complexity of the algorithm. In [50], Vaidyanathan and Nguyen suggested the optimization of a different objective function which can be expressed as a quadratic form in terms of the coefficient vector $\mathbf{b}$. By subjecting $\mathbf{b}$ to a unit norm constraint to avoid trivial solutions, the resulting optimal $\mathbf{b}$ can be obtained via Rayleigh's principle [12] in much the same way that prolate spheroidal wave sequences are obtained [42].

\section{Early Work on Eigenfilters: Low-Pass Eigenfilters}

Vaidyanathan and Nguyen [50] introduced the notion of an "eigenfilter," namely a filter whose coefficients are the components of an eigenvector, by first focusing on designing a low-pass filter. They considered approximating a low-pass filter with passband frequency $\omega_{p}$ and stopband frequency $\omega_{S}$, whose desired response is

$$
D(\omega)= \begin{cases}1, & 0 \leq \omega \leq \omega_{p} \\ 0, & \omega_{S} \leq \omega \leq \pi \\ \text { don't care, } & \omega_{p}<\omega<\omega_{S} .\end{cases}
$$

To approximate $D(\omega)$ as in (12), two quantities were considered in [50], a stopband error measure $\xi_{S}$ and a passband error 
measure $\xi_{p}$. For the stopband error $\xi_{S}$, the mean-squared error of (9) was used, given by

$$
\begin{aligned}
\xi_{S} & \triangleq \frac{1}{\pi} \int_{\omega_{S}}^{\pi}\left[D(\omega)-H_{R}(\omega)\right]^{2} d \omega \\
& =\frac{1}{\pi} \int_{\omega_{S}}^{\pi} \mathbf{b}^{T} \mathbf{c}(\omega) \mathbf{c}^{T}(\omega) \mathbf{b} d \omega=\mathbf{b}^{T} \mathbf{P}_{S} \mathbf{b}
\end{aligned}
$$

where $\mathbf{P}_{S}$ is a real, symmetric, positive definte matrix given by

$$
\mathbf{P}_{S} \triangleq \frac{1}{\pi} \int_{\omega_{S}}^{\pi} \mathbf{c}(\omega) \mathbf{c}^{T}(\omega) d \omega
$$

Unlike the stopband error measure, if the mean-squared error of (9) for the passband error is used, the resulting expression will not be in the form of a quadratic form in terms of the vector $\mathbf{b}$. To bypass this dilemma, a reference frequency was introduced into the design problem and the passband error measure was taken to be the deviation of the amplitude response from its value at the reference frequency. In particular, for $\xi_{p}$, the deviation of $H_{R}(\omega)$ from its value at the reference frequency $\omega=0$ was measured. As $H_{R}(0)=\mathbf{b}^{T} \mathbf{1}$, where $\mathbf{1}$ is an $(M+1) \times 1$ vector of all 1's, $\xi_{p}$ was chosen to be

$$
\begin{aligned}
\xi_{p} & \triangleq \frac{1}{\pi} \int_{0}^{\omega_{p}}\left[H_{R}(0)-H_{R}(\omega)\right]^{2} d \omega \\
& =\frac{1}{\pi} \int_{0}^{\omega_{p}} \mathbf{b}^{T}(\mathbf{1}-\mathbf{c}(\omega))(\mathbf{1}-\mathbf{c}(\omega))^{T} \mathbf{b} d \omega=\mathbf{b}^{T} \mathbf{P}_{p} \mathbf{b}
\end{aligned}
$$

where $\mathbf{P}_{p}$ is a real, symmetric, positive definite matrix given by

$$
\mathbf{P}_{p} \triangleq \frac{1}{\pi} \int_{0}^{\omega_{p}}(\mathbf{1}-\mathbf{c}(\omega))(\mathbf{1}-\mathbf{c}(\omega))^{T} d \omega
$$

To jointly minimize both $\xi_{S}$ and $\xi_{p}$, the authors in [50] considered minimizing a convex combination [12] of the two. In particular, the vector $\mathbf{b}$ was chosen to minimize

$$
\xi \triangleq \alpha \xi_{S}+(1-\alpha) \xi_{p}, 0 \leq \alpha \leq 1
$$

where $\alpha$ is a tradeoff parameter between stopband and passband performances. In light of (13) and (14), $\xi$ in (15) can be expressed as follows:

$$
\xi=\mathbf{b}^{T} \mathbf{P b}, \text { where } \mathbf{P} \triangleq \alpha \mathbf{P}_{S}+(1-\alpha) \mathbf{P}_{p} .
$$

Note that the matrix $\mathbf{P}$ is itself a real, symmetric, positive definite matrix since $0 \leq \alpha \leq 1$. If we impose that $\mathbf{b}$ has unit norm, i.e., $\mathbf{b}^{T} \mathbf{b}=1$, to avoid trivial solutions, then the optimal b which minimizes $\xi$ in (16) is simply the eigenvector corresponding to the minimum eigenvalue $\lambda_{0}$ of $\mathbf{P}$ by Rayleigh's principle [12].

As opposed to the least-squares approach for filter design which requires the computation of a matrix inverse as can be seen from (11), the eigenfilter method only requires the computation of a single eigenvector of a Hermitian positive definite matrix. This eigenvector can be calculated efficiently using the iterative power method [12], [49]. The power method, which is formally used to calculate the largest eigenvalue of a Hermitian positive semidefinite matrix and its corresponding eigenvector, is known to converge quickly when the largest eigenvalue is much larger in magnitude than the next largest eigenvalue. In

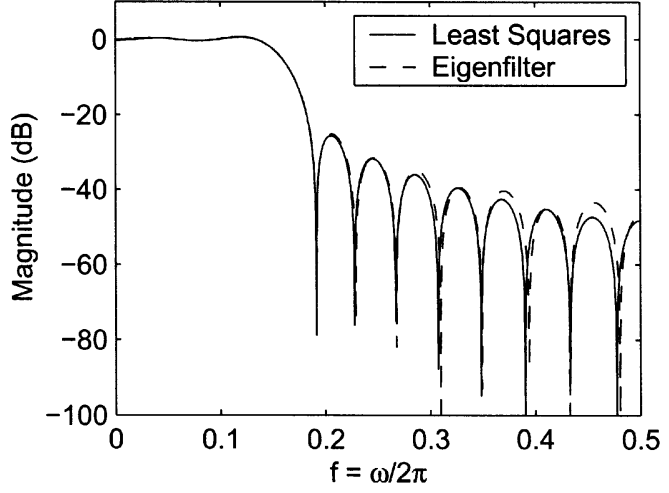

Fig. 2. Magnitude responses obtained using the least-squares approach along with the eigenfilter approach. $\left(\omega_{p}=0.3 \pi, \omega_{S}=0.35 \pi, N=24, W(\omega)=1\right.$, $\alpha=1 / 2)$.

such cases, the complexity of the eigenfilter method is much less than that of the least-squares approach.

In Fig. 2, the magnitude responses using the eigenfilter method as well as the least-squares approach are plotted for a low-pass filter with $\omega_{p}=0.3 \pi$ and $\omega_{S}=0.35 \pi$. Here, the filter order was chosen to be $N=24$ and equal weighing was used for both the passband and stopband (i.e., we chose $W(\omega) \equiv 1$ for the least-squares approach and $\alpha=1 / 2$ for the eigenfilter method). The eigenfilter was rescaled to have unity gain at $\omega=0$. From the plots, it can be seen that the eigenfilter is very similar to the least-squares filter, although the former performs slightly worse in some parts of the stopband. As $N$ increases, the two responses become more and more alike. The close agreement between the least-squares approach and the eigenfilter method, along with the lower complexity of the latter, show the merits of the eigenfilter method.

As mentioned earlier, the tradeoff parameter $\alpha$ controls the performances of the passband and stopband. When $\alpha \approx 1$, most of the design emphasis is on the stopband, and consequently the stopband ripple sizes are smaller than for other values of $\alpha$. A similar scenario holds for the passband when $\alpha \approx 0$.

In addition to designing low-pass filters using the eigenfilter approach, the authors in [50] also showed how to design bandpass and multiband filters with the introduction of different reference frequencies.

1) Incorporating Time- and Frequency-Domain Constraints: Another advantage of the eigenfilter method shown by Vaidyanathan and Nguyen is the ease with which certain time and frequency-domain constraints can be accounted for in the design. In particular, they focused on the time-domain Nyquist constraint [49] and also a frequency-domain flatness constraint. It was shown that even with such constraints, the resulting filter coefficients could be found using the eigenfilter technique.

In [50], the authors first considered the Nyquist constraint. The causal FIR filter $h(n)$ of order $2 M$ we have focused on thus far is said to satisfy the Nyquist constraint [or said to be Nyquist $(K)]$ if [49], [50]

$$
h(K m+M)=C \delta(m)
$$

where $K$ is some positive integer and $C$ is some constant. In other words, the advanced response $h_{a}(n) \triangleq h(n+M)$ be- 
comes an impulse after being decimated by $K$ [49]. Using (8), the coefficients $\left\{b_{n}\right\}$ must satisfy

$$
b_{K m}=C \delta(m), 0 \leq m \leq\left\lfloor\frac{M}{K}\right\rfloor .
$$

As such, the coefficient vector $\mathbf{b}$ is of the form in (18), shown at the bottom of the page. From (16) and (18), it is apparent that the rows and columns of $\mathbf{P}$ whose indices are nonzero multiples of $K$ do not contribute to the overall error $\xi=\mathbf{b}^{T} \mathbf{P b}$. Hence, $\xi$ can be rewritten as

$$
\xi=\widehat{\mathbf{b}}^{T} \widehat{\mathbf{P}} \widehat{\mathbf{b}}
$$

where $\widehat{\mathbf{b}}$ is simply the vector $\mathbf{b}$ of (18) with the zeros removed, as shown in the second equation at the bottom of the page and $\widehat{\mathbf{P}}$ is the matrix obtained by removing the rows and columns of $\mathbf{P}$ whose indices are nonzero multiples of $K$. From (18), the constraint $\mathbf{b}^{T} \mathbf{b}=1$ is equivalent to $\widehat{\mathbf{b}}^{T} \widehat{\mathbf{b}}=1$. Thus, the design problem is to minimize $\xi=\widehat{\mathbf{b}}^{T} \widehat{\mathbf{P}} \widehat{\mathbf{b}}$ subject to $\widehat{\mathbf{b}}^{T} \widehat{\mathbf{b}}=1$, which is the traditional eigenfilter problem, but with the smaller matrix $\widehat{\mathbf{P}}$.

In addition to showing how the Nyquist constraint could be incorporated in the design of eigenfilters, it was also shown how to incorporate flatness constraints in the frequency domain. By definition [49], a frequency response $G\left(e^{j \omega}\right)$ is said to have a degree of flatness of $K$ at the frequency $\omega_{0}$ if we have

$$
\left.\frac{d^{k}}{d \omega^{k}} G\left(e^{j \omega}\right)\right|_{\omega=\omega_{0}}=0,1 \leq k \leq K .
$$

For simplicity, the authors in [50] focused on a flatness constraint at $\omega=0$ and later showed how to accomodate for any arbitrary frequency $\omega_{0}$. Recall that $H_{R}(\omega)$ from (7) is an expansion in terms of the functions $\{\cos \omega n\}_{n=0}^{M}$. Alternatively, $H_{R}(\omega)$ can be expressed in terms of the functions $\left\{\sin ^{2 n}(\omega / 2)\right\}_{n=0}^{M}$, yielding the expansion

$$
H_{R}(\omega)=\sum_{n=0}^{M} d_{n} \sin ^{2 n}\left(\frac{\omega}{2}\right) .
$$

With this expansion, it can easily be shown that the flatness constraints of (19) are satisfied for $G\left(e^{j \omega}\right)=H_{R}(\omega), K=$ $2 L+1$, and $\omega_{0}=0$ iff $d_{k}=0$ for $1 \leq k \leq L$. Thus, if $d_{k}=0$ for $1 \leq k \leq L$, then $H_{R}(\omega)$ has a $2 L+1$ degree of flatness at $\omega=0$. Assuming this to be the case, then by defining the following vectors:

$$
\begin{aligned}
\widehat{\mathbf{d}} & \triangleq\left[\begin{array}{lllll}
d_{0} & d_{L+1} & d_{L+2} & \cdots & d_{M}
\end{array}\right]^{T} \\
\widehat{\mathbf{s}}(\omega) & \triangleq\left[\begin{array}{lllll}
1 & \sin ^{2(L+1)}\left(\frac{\omega}{2}\right) & \sin ^{2(L+2)}\left(\frac{\omega}{2}\right) & \cdots & \sin ^{2 M}\left(\frac{\omega}{2}\right)
\end{array}\right]^{T}
\end{aligned}
$$

we have $H_{R}(\omega)=\widehat{\mathbf{d}}^{T} \widehat{\mathbf{s}}(\omega)$. Note that the vector $\widehat{\mathbf{d}}$ is completely arbitrary and that the flatness constraint is automatically satisfied by construction. In this case, the objective function from
(15) takes on the form $\xi=\widehat{\mathbf{d}}^{T} \mathbf{P} \widehat{\mathbf{d}}$, where $\mathbf{P}$ is as in (16) but with $\mathbf{P}_{S}$ and $\mathbf{P}_{p}$, given by

$$
\begin{aligned}
& \mathbf{P}_{S}=\frac{1}{\pi} \int_{\omega_{S}}^{\pi} \widehat{\mathbf{s}}(\omega) \widehat{\mathbf{s}}^{T}(\omega) d \omega \\
& \mathbf{P}_{p}=\frac{1}{\pi} \int_{0}^{\omega_{p}}(\widehat{\mathbf{1}}-\widehat{\mathbf{s}}(\omega))(\widehat{\mathbf{1}}-\widehat{\mathbf{s}}(\omega))^{T} d \omega
\end{aligned}
$$

where $\widehat{1}=\left[\begin{array}{lllll}1 & 0 & 0 & \cdots & 0\end{array}\right]^{T}$. Under the unit norm constraint $\widehat{\mathbf{d}}^{T} \widehat{\mathbf{d}}=1, \xi$ can be minimized using Rayleigh's principle as before. To generalize this approach to have a $2 L+1$ degree of flatness at any arbitrary frequency $\omega_{0}$, we need only expand $H_{R}(\omega)$ in terms of the functions $\left\{\sin ^{2 n}\left(\omega-\omega_{0} / 2\right)\right\}_{n=0}^{M}$.

2) Iterative Eigenfilter Method for the Design of Equiripple Filters: The McClellan-Parks algorithm [16], which minimizes the $L_{\infty}$ norm of the response error, is widely used for equiripple filter design on account of its rapid convergence and good performance. However, incorporating time- and frequency-domain constraints into the design, such as those from above, is often very difficult. The relative ease with which the eigenfilter method can accomodate such constraints was the primary motivation for Vaidyanathan and Nguyen to develop an iterative eigenfilter technique for designing equiripple filters.

To formulate an iterative technique, the authors in [50] introduced a weight function $W(\omega)$ for the passband and stopband error measures of (14) and (13), respectively, given by

$$
\xi_{p}=\frac{1}{\pi} \int_{0}^{\omega_{p}} W(\omega) e_{p}^{2}(\omega) d \omega, \xi_{S}=\frac{1}{\pi} \int_{\omega_{S}}^{\pi} W(\omega) e_{S}^{2}(\omega) d \omega
$$

where $e_{p}(\omega)=\mathbf{b}^{T}(\mathbf{1}-\mathbf{c}(\omega))$ and $e_{S}(\omega)=\mathbf{b}^{T} \mathbf{c}(\omega)$. For uniform weighting (i.e., $W(\omega) \equiv 1$ ), the errors $e_{p}(\omega)$ and $e_{S}(\omega)$ were observed to be large near the band edges and smaller away from them. As $e_{p}(\omega)$ and $e_{S}(\omega)$ are not known a priori, an iterative procedure to identify the appropriate $W(\omega)$ was suggested based on the above observation. If $e_{p, k}(\omega)$ and $e_{S, k}(\omega)$ denote the passband and stopband error curves after the $k$ th iteration, the weighting function for the $(k+1)$ th iteration was chosen as

$$
W_{k+1}(\omega)= \begin{cases}W_{k}(\omega)\left|e_{p, k}(\omega)\right|, & 0 \leq \omega \leq \omega_{p} \\ W_{k}(\omega)\left|e_{S, k}(\omega)\right|, & \omega_{S} \leq \omega \leq \pi\end{cases}
$$

with the initialization $W_{1}(\omega) \equiv 1$. Here, either $e_{p, k}(\omega)$ and $e_{S, k}(\omega)$ or their envelopes can be used. The iteration is carried out until the magnitude of the difference in the solutions is smaller than a prescribed small constant $\epsilon$. In other words, the iteration is carried out until $\left\|\mathbf{b}_{k+1}-\mathbf{b}_{k}\right\|<\epsilon$.

In Fig. 3, we have plotted the magnitude response of an equiripple filter designed using the iterative eigenfilter method along with that obtained by the Remez exchange algorithm used in the McClellan-Parks program. To compare both methods, we designed a low-pass filter without any time or frequency-domain constraints, as the McClellan-Parks algorithm is incapable of incorporating them. Here, $\omega_{p}=0.3 \pi$

$$
\mathbf{b}=\left[\begin{array}{llllllllllll}
b_{0} & b_{1} & \cdots & b_{K-1} & 0 & b_{K+1} & \cdots & b_{2 K-1} & 0 & b_{2 K+1} & \cdots & \cdots
\end{array}\right]
$$

$$
\widehat{\mathbf{b}} \triangleq\left[\begin{array}{llllllllll}
b_{0} & b_{1} & \cdots & b_{K-1} & b_{K+1} & \cdots & b_{2 K-1} & b_{2 K+1} & \cdots & \cdots
\end{array}\right]
$$




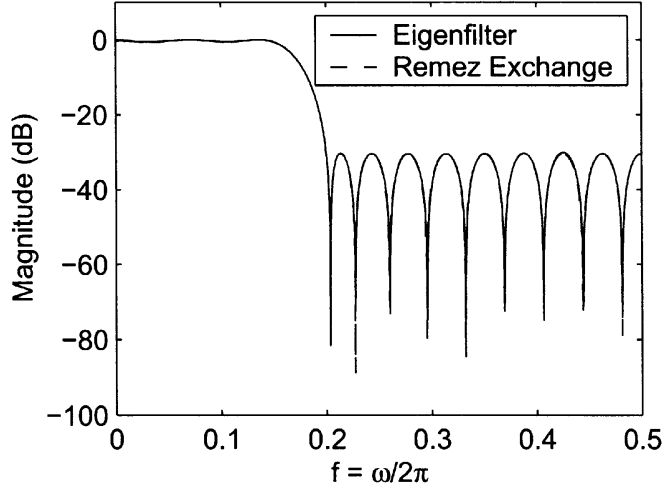

(a)

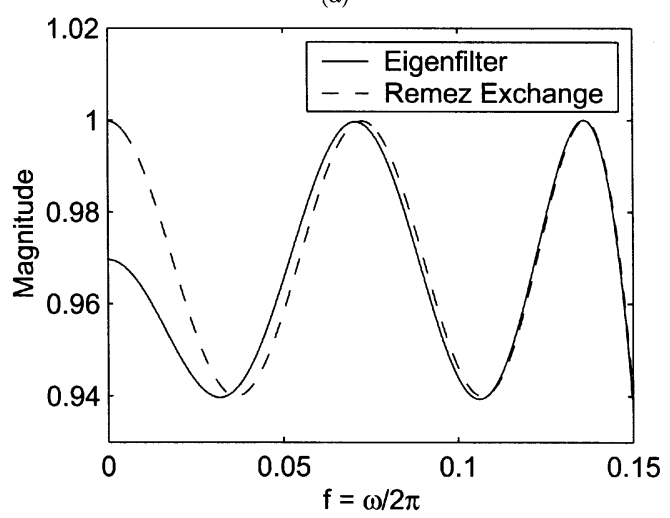

(b)

Fig. 3. Magnitude response of equiripple filters designed using the eigenfilter and Remez exchange algorithms. (a) Magnitude response plot on a decibel scale. (b) Passband details using a nondecibel scale.

and $\omega_{S}=0.4 \pi$, and we chose $N=28$ for both methods. For the eigenfilter method, we chose $\alpha=0.5$. A decibel plot of the magnitude response is shown in Fig. 3(a), while the passband details are shown in Fig. 3(b) on an nondecibel scale. From Fig. 3(a), we see that both methods are in excellent agreement. The eigenfilter is only approximately equiripple since the reference frequency condition at $\omega=0$ prevents this frequency from being an extremal frequency. As such, the eigenfilter exhibits a larger, albeit only slightly larger, $L_{\infty}$ error than the filter designed using the Remez exchange algorithm. Using the methods of the previous subsection, we can create approximately equiripple eigenfilters that satisfy certain time and frequency-domain constraints.

\section{Generalizations of the EIGENFILTER Method}

Since the work of Vaidyanathan and Nguyen [50], many generalizations have been made to the eigenfilter method. In this section, we will focus on several such extensions, including using the eigenfilter method to design arbitrary amplitude and frequency response filters and incorporating general linear constraints into the design. In addition, we will review a recent eigenfilter method based on a total least-squares-error criterion. Then, we will show how the eigenfilter method can be used to design IIR filters including all-pass filters. Finally, we will focus on designing multidimensional FIR filters using the eigenfilter method.

\section{A. Arbitrary Desired Response Eigenfilters}

Nguyen originally considered the problem of using the eigenfilter method to approximate an arbitrary real desired amplitude response $D(\omega)$ using linear-phase FIR filters [20]. Later, the method was generalized to approximating a general complex desired response $D(\omega)$ with a complex coefficient FIR filter [21], [29]. Here, we present the methods of [20], as well as those of [29].

1) Arbitrary Amplitude Response Case: In order to approximate any real desired amplitude response $D(\omega)$ with a linearphase FIR filter using the eigenfilter method, Nguyen [20] considered modifying the least-squares-error criterion (9) by incorporating a reference frequency at which the FIR filter amplitude response $H_{R}(\omega)$ would exactly match $D(\omega)$. More specifically, Nguyen considered minimizing the objective function

$$
\xi \triangleq \frac{1}{\pi} \int_{\mathcal{R}} W(\omega)[\underbrace{\frac{D(\omega)}{D\left(\omega_{0}\right)} H_{R}\left(\omega_{0}\right)-H_{R}(\omega)}_{\epsilon(\omega)}]^{2} d \omega .
$$

(In [20], Nguyen considered the special case $W(\omega) \equiv 1$, but here we include an arbitrary $W(\omega)$ for sake of generality.) Here, $\omega_{0}$ is the reference frequency which is chosen by the designer. Note that the error function $\epsilon(\omega)$ is chosen by construction to be zero at $\omega_{0}$. In [20], $\omega_{0}$ was chosen to be

$$
\omega_{0} \triangleq \underset{\omega \in \partial \mathcal{R}}{\operatorname{argmax}}|D(\omega)|
$$

where $\partial \mathcal{R}$ denotes the boundary of the region $\mathcal{R}$, whereas in [21], $\omega_{0}$ was chosen to be

$$
\omega_{0} \triangleq \underset{\omega \in[0, \pi]}{\operatorname{argmax}}|D(\omega)|
$$

Pei and Shyu [29] showed through a design example that the choice of $\omega_{0}$ had a noticable effect on the overall performance of the resulting filter. From their simulations, it could be seen heuristically that choosing $\omega_{0}$ where $D\left(\omega_{0}\right)$ was large in magnitude yielded better results, thus justifying the choices of $\omega_{0}$ suggested by Nguyen in (23) and (24). Using the fact that $H_{R}(\omega)=$ $\mathbf{b}^{T} \mathbf{c}(\omega)$, it can be shown from (22) that we have the equation shown at the bottom of the page. As before, the matrix $\mathbf{P}$ is a real, symmetric, positive definite matrix. With the usual unit norm constraint $\mathbf{b}^{T} \mathbf{b}=1$ on $\mathbf{b}$, the optimal coefficients for $H_{R}(\omega)$ can be obtained using the eigenfilter method as before. It should be noted that after the optimal $\mathbf{b}$ is found, the solution must be scaled in order to satisfy the reference frequency condition that $H_{R}\left(\omega_{0}\right)=D\left(\omega_{0}\right)$, which is equivalent to $\mathbf{b}^{T} \mathbf{c}\left(\omega_{0}\right)=D\left(\omega_{0}\right)$.

$$
\xi=\mathbf{b}^{T} \mathbf{P} \mathbf{b}, \text { where } \mathbf{P}=\frac{1}{\pi} \int_{\mathcal{R}} W(\omega)\left[\frac{D(\omega)}{D\left(\omega_{0}\right)} \mathbf{c}\left(\omega_{0}\right)-\mathbf{c}(\omega)\right]\left[\frac{D(\omega)}{D\left(\omega_{0}\right)} \mathbf{c}\left(\omega_{0}\right)-\mathbf{c}(\omega)\right]^{T} d \omega
$$


2) Arbitrary Desired Response Case: Nguyen as well as Pei and Shyu independently developed different methods to design a complex coefficient FIR filter to approximate a generally complex valued desired response using the eigenfilter method [29], [21]. Here, we present the method of Pei and Shyu [29].

Let $h(n)$ be a causal FIR filter of length $N$, so that $H(z)=$ $\sum_{n=0}^{N-1} h(n) z^{-n}$. Defining the $N \times 1$ vectors

$$
\begin{aligned}
\mathbf{h} & \triangleq\left[\begin{array}{llll}
h(0) & h(1) & \cdots & h(N-1)
\end{array}\right]^{T} \\
\mathbf{e}(z) & \triangleq\left[\begin{array}{llll}
1 & z^{-1} & \cdots & z^{-(N-1)}
\end{array}\right]^{T}
\end{aligned}
$$

we clearly have $H(z)=\mathbf{h}^{T} \mathbf{e}(z)$. To develop an eigenfilterbased method for approximating a complex valued desired response $D(\omega)$, Pei and Shyu considered modifying the following least-squares objective function,

$$
\xi_{\mathrm{WLS}} \triangleq \frac{1}{2 \pi} \int_{\mathcal{R}} W(\omega)\left|D(\omega)-H\left(e^{j \omega}\right)\right|^{2} d \omega
$$

in order to express it as a quadratic form in terms of the vector $\mathbf{h}$. (In [29], the authors did not include the weight function $W(\omega)$ or the constant $1 /(2 \pi)$ in their analysis. We have decided to include both quantities for sake of completeness.) The frequency region $\mathcal{R}$ is now a subset of the interval $[0,2 \pi]$ and the desired response $D(\omega)$ is allowed to be complex. Similarly to what was done in the previous section, to pose the design problem as an eigenfilter problem, Pei and Shyu modified (26) to incorporate a reference frequency $\omega_{0}$ at which the frequency response of the filter would match the desired response. In particular, they considered minimizing the objective

$$
\xi \triangleq \frac{1}{2 \pi} \int_{\mathcal{R}} W(\omega)\left|\frac{D(\omega)}{D\left(\omega_{0}\right)} H\left(e^{j \omega_{0}}\right)-H\left(e^{j \omega}\right)\right|^{2} d \omega
$$

subject to the unit norm constraint $\mathbf{h}^{\dagger} \mathbf{h}=1$. Using $H(z)=$ $\mathbf{h}^{T} \mathbf{e}(z)$, it can be shown that we have the equation shown at the bottom of the page. Here, the $N \times N$ matrix $\mathbf{P}$ is a complex-valued Hermitian, positive definite matrix. Under the unit norm constraint $\mathbf{h}^{\dagger} \mathbf{h}=1$, the optimal filter coefficients can be found using Rayleigh's principle, which applies for any Hermitian matrix, be it real or complex. After obtaining the optimal coefficients, the resulting filter must be scaled in order to satisfy the reference frequency condition $H\left(e^{j \omega_{0}}\right)=\mathbf{h}^{T} \mathbf{e}\left(e^{j \omega_{0}}\right)=$ $D\left(\omega_{0}\right)$. In [21], Nguyen solved the same problem, but decoupled $h(n)$ into real and imaginary parts and then expressed the objective as a quadratic form in terms of the vector of real and imaginary parts of $h(n)$. Whereas Pei and Shyu's method involved an $N \times N$ complex matrix, Nguyen's method involved a $2 N \times 2 N$ real matrix.

As an example, suppose that the magnitude and group delay of the desired response $D(\omega)$ are as shown in Fig. 4(a) and (b), respectively. (This example was considered by Nguyen [21].) Recall that the group delay $\tau(\omega)$ is the negative of the derivative of the phase with respect to $\omega$ [49]. Namely, if $D(\omega)=$

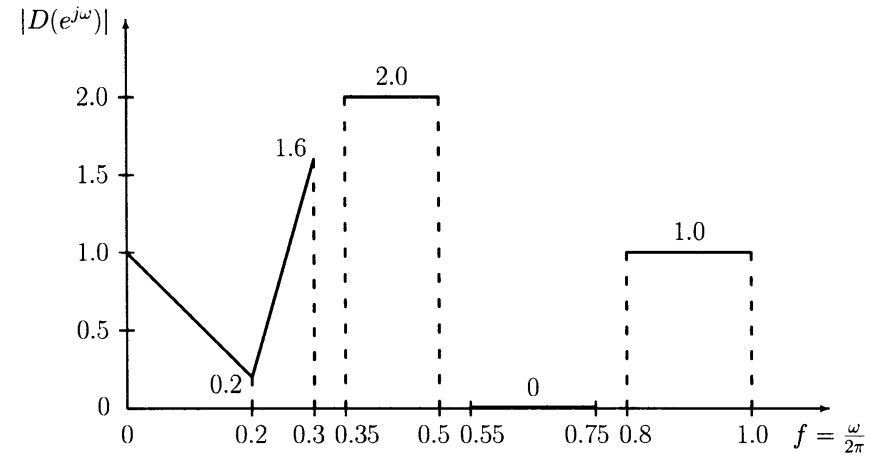

(a)

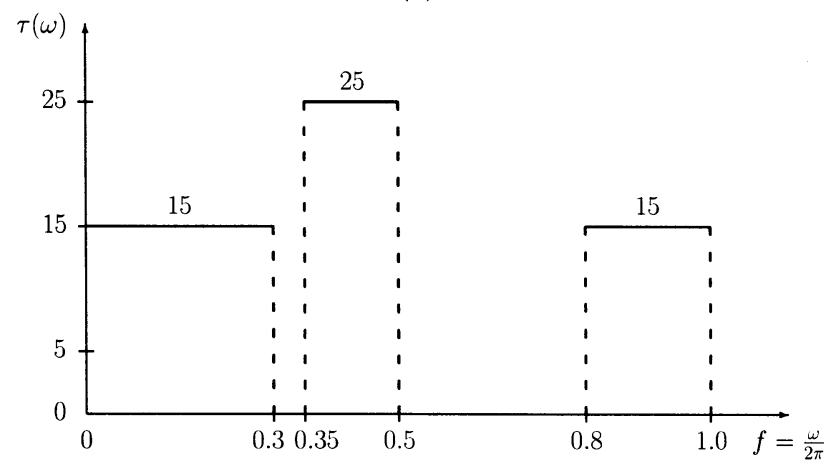

(b)

Fig. 4. (a) Desired magnitude response. (b) Desired group delay response.

$|D(\omega)| e^{j \phi(\omega)}$, then $\tau(\omega)=-d \phi(\omega) / d \omega$. With a filter length of $N=50$, a reference frequency of $\omega_{0}=0.85 \pi$, and uniform weighting, the resulting eigenfilter magnitude and group delay responses are shown in Fig. 5(a) and (b). As we can see, the eigenfilter designed here yielded a good fit to the desired response in the frequency regions of interest.

\section{B. Incorporating General Linear Constraints Using the Eigenfilter Approach}

Often times in the design of FIR filters, it is desired that the filter coefficients satisfy a general linear constraint. For example, if we consider the complex coefficient FIR filter from Section III-A-2, suppose that in addition to choosing $H\left(e^{j \omega}\right)$ to approximate $D(\omega)$, we would like $H\left(e^{j \omega}\right)$ as well as its first $L$ derivatives to match those of $D(\omega)$ at some reference frequency $\omega_{0}$. Namely, we wish to choose $H\left(e^{j \omega}\right)$ to satisfy

$$
\left.\frac{d^{k} H\left(e^{j \omega}\right)}{d \omega^{k}}\right|_{\omega=\omega_{0}}=\left.\frac{d^{k} D(\omega)}{d \omega^{k}}\right|_{\omega=\omega_{0}}, 0 \leq k \leq L
$$

Note that this is similar to the flatness constraints of (19) considered in Section II-C-1. As we have $H\left(e^{j \omega}\right)=\mathbf{h}^{T} \mathbf{e}\left(e^{j \omega}\right)=$ $\mathbf{e}^{T}\left(e^{j \omega}\right) \mathbf{h}$, the constraints in (28) can be rewritten in the form

$$
\mathrm{Ch}=\mathrm{d}
$$

$$
\xi=\mathbf{h}^{\dagger} \mathbf{P h}, \text { where } \mathbf{P}=\frac{1}{2 \pi} \int_{\mathcal{R}} W(\omega)\left[\frac{D(\omega)}{D\left(\omega_{0}\right)} \mathbf{e}\left(e^{j \omega_{0}}\right)-\mathbf{e}\left(e^{j \omega}\right)\right]^{*}\left[\frac{D(\omega)}{D\left(\omega_{0}\right)} \mathbf{e}\left(e^{j \omega_{0}}\right)-\mathbf{e}\left(e^{j \omega}\right)\right]^{T} d \omega
$$




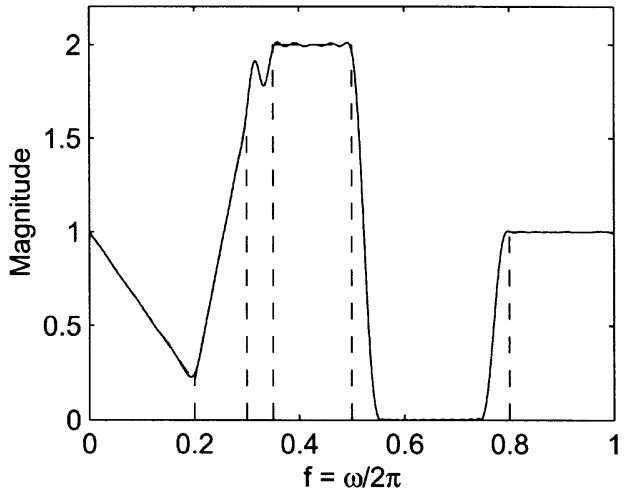

(a)

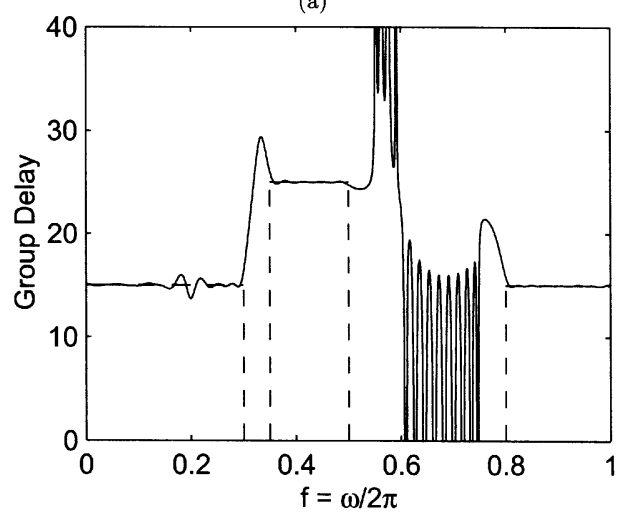

(b)

Fig. 5. (a) Magnitude response of the complex eigenfilter. (b) Group delay response. $\left(N=50, \omega_{0}=0.85 \pi\right)$.

where $\mathbf{C}$ is a $(L+1) \times N$ matrix and $\mathbf{d}$ is a $(L+1) \times 1$ column vector given by

$$
\mathbf{C}=\left[\begin{array}{c}
\mathbf{e}^{T}\left(e^{j \omega_{0}}\right) \\
\frac{d}{d \omega} \mathbf{e}^{T}\left(e^{j \omega_{0}}\right) \\
\vdots \\
\frac{d^{K}}{d \omega^{K}} \mathbf{e}^{T}\left(e^{j \omega_{0}}\right)
\end{array}\right], \mathbf{d}=\left[\begin{array}{c}
D\left(\omega_{0}\right) \\
\frac{d}{d \omega} D\left(\omega_{0}\right) \\
\vdots \\
\frac{d^{K}}{d \omega^{K}} D\left(\omega_{0}\right)
\end{array}\right]
$$

Note that the constraint equation in (29) is linear with respect to the coefficient vector $\mathbf{h}$. Incorporating general linear constraints as in (29) using the eigenfilter approach was first considered by Chen [10] for the special case where $\mathbf{d}=\mathbf{0}$ and later generalized by Pei et al. [37] for $\mathbf{d} \neq 0$. We present the results of [37].

Recall that for the complex case, the eigenfilter problem is typically formulated as follows:

$$
\text { Minimize } \xi=\mathbf{h}^{\dagger} \mathbf{P h} \text {, subject to } \mathbf{h}^{\dagger} \mathbf{h}=1
$$

where $\mathbf{P}$ is some Hermitian matrix. It should be noted that the constraint here is really just included to prevent trivial solutions from occuring. Typically after the optimal $\mathbf{h}$ is found, the true coefficient vector $\widehat{\mathbf{h}}$ that we use is the optimal $\mathbf{h}$ scaled by a factor in order to satisfy the reference frequency condition. In other words, we use $\widehat{\mathbf{h}}=K \mathbf{h}$, where $K$ is chosen such that we have $\mathbf{e}^{T}\left(e^{j \omega_{0}}\right) \widehat{\mathbf{h}}=D\left(\omega_{0}\right)$ (which implies $K=$ $\left.D\left(\omega_{0}\right) / \mathbf{e}^{T}\left(e^{j \omega_{0}}\right) \mathbf{h}\right)$. By expressing $\xi$ as a Rayleigh quotient
[12] in terms of the true coefficient vector $\widehat{\mathbf{h}}$, the design problem becomes

$$
\text { Minimize } \xi=\frac{\widehat{\mathbf{h}}^{\dagger} \mathbf{P} \widehat{\mathbf{h}}}{\widehat{\mathbf{h}}^{\dagger} \widehat{\mathbf{h}}}
$$

over all nonzero vectors $\widehat{\mathbf{h}}$. By Rayleigh's principle, the minimum value of $\xi$ is the smallest eigenvalue $\lambda_{0}$ of $\mathbf{P}$ which occurs iff $\widehat{\mathbf{h}}$ is in the eigenspace corresponding to $\lambda_{0}$. Note that here the length of $\hat{\mathbf{h}}$ is arbitrary. In the eigenfilter design problem, the length of $\hat{\mathbf{h}}$ is then chosen to satisfy the condition

$$
\mathbf{e}^{T}\left(e^{j \omega_{0}}\right) \widehat{\mathbf{h}}=D\left(\omega_{0}\right) \Longleftrightarrow\left(\frac{\mathbf{e}^{T}\left(e^{j \omega_{0}}\right)}{D\left(\omega_{0}\right)}\right) \widehat{\mathbf{h}}=1 .
$$

If $\widehat{\mathbf{h}}$ must satisfy linear constraints of the form given in (29), the design problem becomes

$$
\text { Minimize } \xi=\frac{\widehat{\mathbf{h}}^{\dagger} \mathbf{P h}}{\widehat{\mathbf{h}}^{\dagger} \widehat{\mathbf{h}}} \text {, subject to } \mathbf{C} \widehat{\mathbf{h}}=\mathbf{d} \text {. }
$$

To solve this optimization problem, Pei, Tseng, and Yang [37] used the equivalent form of the reference frequency condition of (30) to express the linear constraint equation $\mathbf{C h}=\mathbf{d}$ in a more simplified form. Using (30), the linear constraint equation can be expressed as follows:

$$
\widehat{\mathbf{C}} \widehat{\mathbf{h}}=\mathbf{0} \text { where } \widehat{\mathbf{C}} \triangleq \mathbf{C}-\frac{\mathbf{d e}^{T}\left(e^{j \omega_{0}}\right)}{D\left(\omega_{0}\right)} .
$$

Hence, the filter design problem becomes

$$
\text { Minimize } \xi=\frac{\widehat{\mathbf{h}}^{\dagger} \mathbf{P} \widehat{\mathbf{h}}}{\widehat{\mathbf{h}}^{\dagger} \widehat{\mathbf{h}}}, \text { subject to } \widehat{\mathbf{C}} \widehat{\mathbf{h}}=\mathbf{0} \text {. }
$$

The key to solving this constrained minimization problem is to note that $\widehat{\mathbf{C}} \widehat{\mathbf{h}}=\mathbf{0}$ iff $\widehat{\mathbf{h}}$ lies in the null space of $\widehat{\mathbf{C}}$ [12]. Any such $\widehat{\mathbf{h}}$ can be expressed as $\widehat{\mathbf{h}}=\mathbf{U a}$, where $\mathbf{U}$ is a rectangular unitary matrix (i.e., $\mathbf{U}^{\dagger} \mathbf{U}=\mathbf{I}$ ) whose columns form an orthonormal basis for the null space of $\widehat{\mathbf{C}}$ and $\mathbf{a}$ is any arbitrary vector [12]. With this decomposition of $\widehat{\mathbf{h}}$, the design problem becomes

$$
\text { Minimize } \xi=\frac{\mathbf{a}^{\dagger} \mathbf{U}^{\dagger} \mathbf{P U a}}{\mathbf{a}^{\dagger} \mathbf{U}^{\dagger} \mathbf{U} \mathbf{a}}=\frac{\mathbf{a}^{\dagger} \widehat{\mathbf{P}} \mathbf{a}}{\mathbf{a}^{\dagger} \mathbf{a}} \text { where } \widehat{\mathbf{P}} \triangleq \mathbf{U}^{\dagger} \mathbf{P U}
$$

over all nonzero vectors $\mathbf{a}$. As $\widehat{\mathbf{P}}$ is clearly Hermitian, we can use Rayleigh's principle to find the optimal a and then use $\widehat{\mathbf{h}}=\mathbf{U a}$ to find the optimal $\widehat{\mathbf{h}}$. Here, the length of a must be chosen to satisfy the reference frequency condition of (30). Simulation results in [37] using the derivative constraints of (28) showed the usefulness of this eigenfilter technique for designing filters with generalized linear constraints.

\section{Eigenfilter Method Based on a Total Least-Squares-Error Criterion}

Most eigenfilter techniques arose by altering the least-squares-error criterion of (10) or (26) in order to express it as a quadratic form in terms of the filter coefficient vector. As mentioned earlier, this was done by introducing a reference frequency condition into the objective. Recently [38], Pei and Tseng suggested a novel modification to the 
least-squares objective based on a total least-squares error criterion. The resulting objective is still in the form of a Rayleigh quotient in terms of the filter coefficient vector, and so the optimal vector can be found using Rayleigh's principle as before. One advantage of the total least-squares eigenfilter objective over the traditional ones of (22) and (27) is that the former does not require a reference frequency. Another advantage is that for low filter orders, this method has been shown through simulations to come closer to the desired least-squares solution than the conventional eigenfilter method. In addition, for high filter orders, it has been shown to be less susceptible to numerical inaccuracies than the traditional least-squares method which requires matrix inversion.

To properly introduce the total least-square eigenfilter method, we first briefly review the principle of total least-squares. A more thorough description of this principle is provided in [44, pp. 533-535]. In many typical linear modeling problems, given a set of data in the form of a $K \times L$ matrix $\mathbf{X}$, a linear model of the form $\mathbf{X h}$ (where $\mathbf{h}$ is an $L \times 1$ vector) is formed to best approximate a desired $K \times 1$ vector $\mathbf{d}$ in some sense. For the traditional least-squares method, $\mathbf{h}$ is chosen to minimize the squared magnitude of the error

$$
\epsilon=\mathrm{d}-\mathrm{Xh} \Longleftrightarrow \mathrm{Xh}=\mathrm{d}-\boldsymbol{\epsilon}
$$

Namely, $\mathbf{h}$ is chosen to minimize $\xi_{\mathrm{LS}} \triangleq\|\epsilon\|^{2}$. An implicit assumption lbfrom the second equation in (31) is that the error is associated with the desired vector $\mathbf{d}$. In the total least-squares method, an error is not only associated with the desired vector $\mathbf{d}$, but also with the data matrix $\mathbf{X}$. If $\boldsymbol{\Delta}$ is a $K \times L$ matrix representing the error in the matrix $\mathbf{X}$, then the error equation $\mathrm{lbfrom}$ (31) becomes

$$
(\mathbf{X}-\boldsymbol{\Delta}) \mathbf{h}=\mathbf{d}-\boldsymbol{\epsilon}
$$

For the total least-squares method, the vector $\mathbf{h}$ is chosen to minimize the "total" error in both $\mathbf{d}$ and $\mathbf{X}$. In particular, $\mathbf{h}$ is chosen to minimize the objective

$$
\xi_{\mathrm{TLS}} \triangleq\left\|\boldsymbol{\epsilon}_{\mathrm{T}}\right\|_{F}^{2}=\operatorname{Tr}\left[\boldsymbol{\epsilon}_{\mathrm{T}}^{\dagger} \boldsymbol{\epsilon}_{\mathrm{T}}\right], \text { where } \boldsymbol{\epsilon}_{\mathrm{T}} \triangleq\left[\begin{array}{ll}
\boldsymbol{\Delta} & \boldsymbol{\epsilon}
\end{array}\right] .
$$

The $K \times(L+1)$ matrix $\epsilon_{\mathrm{T}}$ represents the error in both the data and desired response. From (32), we get

$$
\left(\widehat{\mathbf{X}}-\boldsymbol{\epsilon}_{\mathrm{T}}\right) \widehat{\mathbf{h}}=\mathbf{0}, \text { where } \widehat{\mathbf{X}} \triangleq\left[\begin{array}{ll}
\mathbf{X} & \mathbf{d}
\end{array}\right] \text {, and } \widehat{\mathbf{h}} \triangleq\left[\begin{array}{c}
\mathbf{h} \\
-1
\end{array}\right] \text {. }
$$

It can be shown [44], that the minimum value of $\xi_{\text {TLS }}$ in (33) is $\lambda_{0}$, where $\lambda_{0}$ is the smallest eigenvalue of $\widehat{\mathbf{X}} \dagger \widehat{\mathbf{X}}$. Furthermore, $\xi_{\text {TLS }}=\lambda_{0}$ iff $\widehat{\mathbf{h}}=K \mathbf{v}_{0}$, where $\mathbf{v}_{0}$ is any vector in the eigenspace corresponding to $\lambda_{0}$, and $K$ is a constant chosen to make the last component of $\widehat{\mathbf{h}}$ equal -1 (assuming that such a constant exists). Because of this, from Rayleigh's principle, an alternative total least-squares-error criterion can be chosen as

$$
\xi_{\mathrm{TLS}} \triangleq \frac{\widehat{\mathbf{h}}^{\dagger} \mathbf{P} \widehat{\mathbf{h}}}{\widehat{\mathbf{h}}^{\dagger} \hat{\mathbf{h}}}, \text { where } \mathbf{P} \triangleq \widehat{\mathbf{X}}^{\dagger} \widehat{\mathbf{X}}=\left[\begin{array}{cc}
\mathbf{X}^{\dagger} \mathbf{X} & \mathbf{X}^{\dagger} \mathbf{d} \\
\mathbf{d}^{\dagger} \mathbf{X} & \mathbf{d}^{\dagger} \mathbf{d}
\end{array}\right]
$$

There is an interesting geometric interpretation between the total least-squares objective $\xi_{\text {TLS }}$ lbfrom (34) and the leastsquares objective $\xi_{\mathrm{LS}}=\|\boldsymbol{\epsilon}\|^{2}$, where $\boldsymbol{\epsilon}$ is as in (31). First note

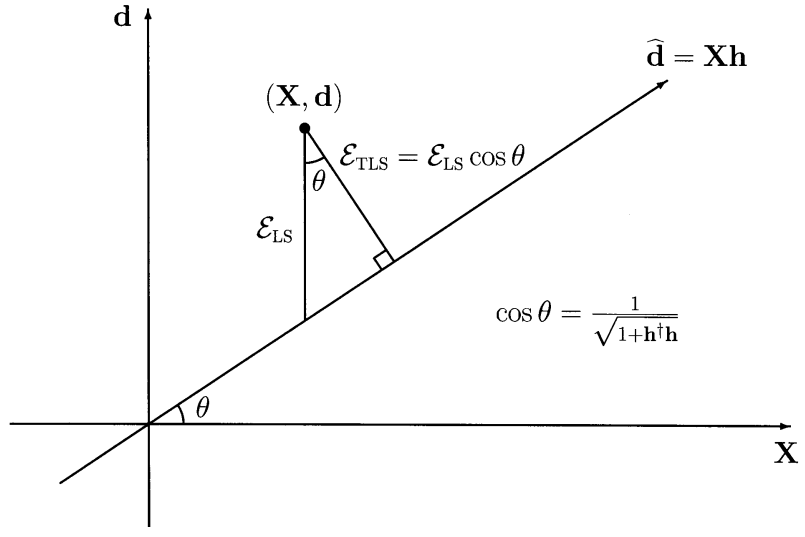

Fig. 6. Geometric interpretation of the least-squares and total least-squares problem.

that it can easily be shown that $\xi_{\mathrm{LS}}=\widehat{\mathbf{h}}^{\dagger} \mathbf{P} \widehat{\mathbf{h}}$. If we define $\mathcal{E}_{\mathrm{LS}} \triangleq \sqrt{\xi_{\mathrm{LS}}}$ and $\mathcal{E}_{\mathrm{TLS}} \triangleq \sqrt{\xi_{\mathrm{TLS}}}$, then clearly we have

$$
\mathcal{E}_{\mathrm{TLS}}=\frac{\mathcal{E}_{\mathrm{LS}}}{\sqrt{1+\mathbf{h}^{\dagger} \mathbf{h}}} \text {. }
$$

If $\widehat{\mathbf{d}}$ is defined as the linear approximation to d, i.e., $\widehat{\mathbf{d}} \triangleq \mathbf{X h}$, then $\mathcal{E}_{\mathrm{LS}}$ represents the length of the path $\mathbf{d}-\widehat{\mathbf{d}}$, whereas $\mathcal{E}_{\mathrm{TLS}}$ represents the minimum distance between $\mathbf{d}$ and the hyperplane $\mathrm{Xh}$, the path of which lies in a direction normal to the hyperplane. These paths are always separated by an angle of $\theta \mathrm{lbfrom}$ each other, where $\cos \theta=1 / \sqrt{1+\mathbf{h}^{\dagger} \mathbf{h}}$. This is shown Ibfor the one-dimensional case in Fig. 6.

To derive the total least-squares eigenfilter objective function, consider the complex least-squares-error criterion of (26). Note that it is of the form

where

$$
\xi_{\mathrm{WLS}}=\frac{1}{2 \pi} \int_{\mathcal{R}} W(\omega)\left(\mathcal{E}_{\mathrm{LS}}(\omega)\right)^{2} d \omega
$$

$$
\mathcal{E}_{\mathrm{LS}}(\omega) \triangleq\left|D(\omega)-\mathbf{h}^{T} \mathbf{e}\left(e^{j \omega}\right)\right| .
$$

(In [38], Pei and Tseng analyzed the real least-squares-error criterion of (10). We have opted to focus on the complex least-squares criterion for sake of generality and notational uniformity.) Note that $\mathcal{E}_{\mathrm{LS}}(\omega)$ is essentially the same quantity $\mathcal{E}_{\mathrm{LS}}$ analyzed above, the only difference being that it depends on the variable $\omega$. From (35) and (36), it is clear that an appropriate total least-squares-error criterion is given as follows:

$$
\xi_{\mathrm{TLS}} \triangleq \frac{1}{2 \pi} \int_{\mathcal{R}} W(\omega)\left(\mathcal{E}_{\mathrm{TLS}}(\omega)\right)^{2} d \omega
$$

where

$$
\mathcal{E}_{\mathrm{TLS}}(\omega) \triangleq \frac{\mathcal{E}_{\mathrm{LS}}(\omega)}{\sqrt{1+\mathbf{h}^{\dagger} \mathbf{h}}} .
$$

Using (36), (37) becomes

$$
\xi_{\mathrm{TLS}}=\frac{1}{2 \pi} \int_{\mathcal{R}} W(\omega) \frac{\left|D(\omega)-\mathbf{h}^{T} \mathbf{e}\left(e^{j \omega}\right)\right|^{2}}{1+\mathbf{h}^{\dagger} \mathbf{h}} d \omega
$$

which can be expressed in the form

$$
\xi_{\mathrm{TLS}}=\frac{\widehat{\mathbf{h}}^{\dagger} \mathbf{P} \widehat{\mathbf{h}}}{\widehat{\mathbf{h}}^{\dagger} \widehat{\mathbf{h}}}, \text { where } \widehat{\mathbf{h}} \triangleq\left[\begin{array}{c}
\mathbf{h} \\
-1
\end{array}\right] .
$$




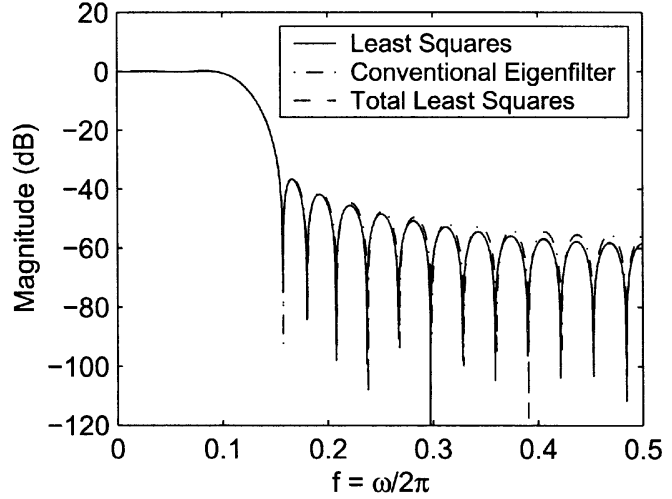

(a)

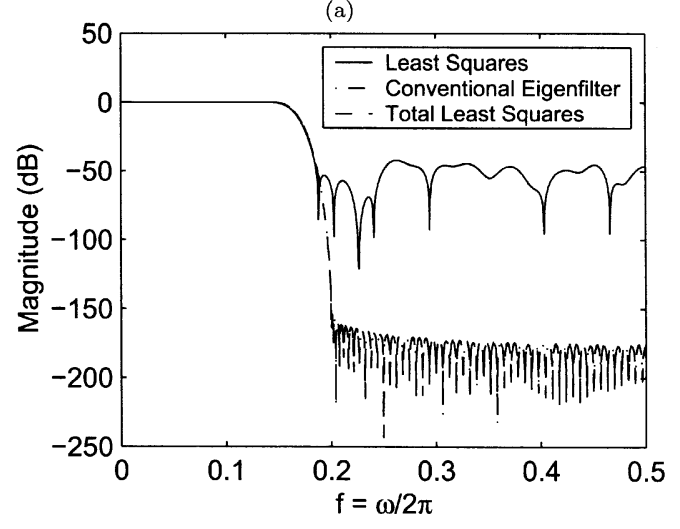

(b)

Fig. 7. (a) Magnitude responses of several low order low-pass filters. $(N=$ $32, \omega_{p}=0.2 \pi, \omega_{S}=0.3 \pi$ ) (b) Magnitude responses of several high order low-pass filters. $\left(N=148, \omega_{p}=0.25 \pi, \omega_{S}=0.4 \pi\right)$.

Here, $\mathbf{P}$ is an $(N+1) \times(N+1)$ matrix of the form

$$
\mathbf{P}=\left[\begin{array}{cc}
\mathbf{A} & \mathbf{b} \\
\mathbf{b} & c
\end{array}\right]
$$

where $\mathbf{A}, \mathbf{b}$, and $c$ are, respectively, the $N \times N, N \times 1$, and $1 \times 1$ quantities

$$
\begin{aligned}
\mathbf{A} & =\frac{1}{2 \pi} \int_{\mathcal{R}} W(\omega) \mathbf{e}^{*}\left(e^{j \omega}\right) \mathbf{e}^{T}\left(e^{j \omega}\right) d \omega \\
\mathbf{b} & =\frac{1}{2 \pi} \int_{\mathcal{R}} W(\omega) D(\omega) \mathbf{e}^{*}\left(e^{j \omega}\right) d \omega \\
c & =\frac{1}{2 \pi} \int_{\mathcal{R}} W(\omega)|D(\omega)|^{2} d \omega .
\end{aligned}
$$

Thus, we have expressed $\xi_{\text {TLS }}$ as a Rayleigh quotient in terms of the vector $\widehat{\mathbf{h}}$. Note the similarities between the matrix $\mathbf{P}$ from (39) and that from (34). By Rayleigh's principle, we can find the optimum $\widehat{\mathbf{h}}$ and thus $\mathbf{h}$. To obtain the proper $\mathbf{h}$, we must scale the optimum $\widehat{\mathbf{h}}$ to satisfy (38).

The merits of the total least-squares eigenfilter method are best seen via simulations. Following an example from Pei and Tseng [38], in Fig. 7(a) and (b), we have plotted the magnitude response of several low-pass filters designed using the traditional least-squares method, the conventional eigenfilter method (which requires a reference frequency), and the total least-squares eigenfilter method. The filters shown in Fig. 7(a) are low order with parameters $N=32, \omega_{p}=0.2 \pi$, and $\omega_{S}=0.3 \pi$. For the conventional eigenfilter method, the reference frequency was chosen to be $\omega_{0}=0$. Though difficult to see, the total least-squares eigenfilter is noticably closer to the optimal least-squares filter than the conventional eigenfilter. In fact, it is indistinguishable from the least-squares filter. Furthermore, the design complexity of the total least-squares eigenfilter is much less than that of the least-squares filter, which requires matrix inversion. The filters in Fig. 7(b) are high order with $N=148, \omega_{p}=0.25 \pi$, and $\omega_{S}=0.4 \pi$ $\left(\omega_{0}=0\right)$. It can be observed that both eigenfilter methods performed about the same, whereas the least-squares filter yielded a poor response. The reason for this is that the matrix which needed to be inverted for the least-squares method was extremely ill-conditioned. For this example, the condition number was $1.7012 \times 10^{14}$. Both eigenfilter methods, as they do not require matrix inversion, are much less susceptible to numerical inaccuracies caused by ill-conditioned matrices than filters designed using the least-squares method. This example serves to show the merits of the total least-squares eigenfilter method for both low order filters as well as high order ones. An extension of the total least-squares method was recently proposed by Zhang and Chen [54] which asymptotically approaches the conventional least-squares solution.

\section{Designing IIR Filters Using the Eigenfilter Method}

1) Design of All-Pass Filters: Thus far, we only focused on designing FIR filters using the eigenfilter method. Several methods for designing IIR filters have also been considered in the literature. The first to consider this problem were Laakso et al. [14] for the design of all-pass phase compensators. Pei and Shyu [33], as well as Nguyen et al. [22], later suggested alternative design methods for these filters. Recently, Zhang and Iwakura [55] considered the design of phase equiripple all-pass filters based on an eigenfilter method. They showed that the optimality criterion for the phase error in the Chebyshev sense could be posed as a generalized eigenvalue problem and proposed an iterative eigenfilter method to design equiripple phase responses. Here, we focus on the method of Pei and Shyu in [33], which is optimal with respect to its error criterion, which is a modification of the phase error $L_{2}$ norm. In contrast, the methods proposed in [14] and [22] are only approximations to the phase error norm.

The transfer function of a causal $N$ th order all-pass function is of the form [49]

$$
\begin{aligned}
& H(z)=\frac{z^{-N} \widetilde{A}(z)}{A(z)}, \text { where } A(z)=\sum_{n=0}^{N} a_{n} z^{-n} \\
& \quad \text { and } \widetilde{A}(z) \triangleq A^{*}\left(\frac{1}{z^{*}}\right) .
\end{aligned}
$$

Here, we will focus on real coefficient all-pass functions for which $a_{n}$ is real for all $n$. In this case, $H(z)$ is of the form $H(z)=z^{-N} A\left(z^{-1}\right) / A(z)$. By construction, the frequency response of $H(z)$ is of unit magnitude, i.e., $\left|H\left(e^{j \omega}\right)\right| \equiv 1$. Hence, the only design freedom present is in the selection of the phase of $H\left(e^{j \omega}\right)$. The phase of $H\left(e^{j \omega}\right)$, which we denote here by $\phi_{H}(\omega)$, is of the form

$$
\phi_{H}(\omega)=-N \omega-2 \phi_{A}(\omega)
$$


where $\phi_{A}(\omega)$ is the phase of $A\left(e^{j \omega}\right)$ given by

$$
\phi_{A}(\omega)=-\arctan \left(\frac{\sum_{k=0}^{N} a_{k} \sin (k \omega)}{\sum_{k=0}^{N} a_{k} \cos (k \omega)}\right) .
$$

Let $\phi_{H, \mathrm{des}}(\omega)$ and $\phi_{A, \mathrm{des}}(\omega)$ denote the desired phase responses for the all-pass filter $H(z)$ and its corresponding denomiator polynomial $A(z)$. From (40), we have $\phi_{H, \text { des }}(\omega)=-N \omega-2 \phi_{A, \operatorname{des}}(\omega)$. Pei and Shyu [33] considered choosing $A(z)$ to match $\phi_{A \text {,des }}(\omega)$, which can be obtained from $\phi_{H, \text { des }}(\omega)$ using the above formula.

In obtaining a criterion to choose the coefficients of the polynomial $A(z)$, Pei and Shyu noted that if $\phi_{A}(\omega)$ was approximately equal to $\phi_{A \text {,des }}(\omega)$, then heuristically we would have

$$
\begin{aligned}
& \left(\sum_{k=0}^{N} a_{k} \cos (k \omega)\right) \sin \left(\phi_{A, \mathrm{des}}(\omega)\right) \\
& \quad-\left(\sum_{k=0}^{N} a_{k} \cos (k \omega)\right) \tan \left(\phi_{A}(\omega)\right) \cos \left(\phi_{A, \text { des }}(\omega)\right) \approx 0 .
\end{aligned}
$$

Equivalently, we would have, using (41)

$$
\begin{aligned}
& \sum_{k=0}^{N} a_{k}\left(\sin \left(\phi_{A, \mathrm{des}}(\omega)\right) \cos (k \omega)+\cos \left(\phi_{A, \mathrm{des}}(\omega)\right) \sin (k \omega)\right) \\
&=\sum_{k=0}^{N} a_{k} \sin \left(\phi_{A, \operatorname{des}}(\omega)+k \omega\right) \approx 0 .
\end{aligned}
$$

Defining the vectors shown at the bottom of the page, then we would have approximately $\mathbf{a}^{T} \mathbf{s}(\omega) \approx 0$ for all $\omega$. To choose $\mathbf{a}$ to ensure this condition as best as possible, Pei and Shyu [33] considered minimizing a mean-squared-error measure of the form

$$
\xi=\frac{1}{\pi} \int_{\mathcal{R}} W(\omega)\left(\mathbf{a}^{T} \mathbf{s}(\omega)\right)^{2} d \omega
$$

subject to a unit norm constraint on a (namely $\mathbf{a}^{T} \mathbf{a}=1$ ). Since the "desired response" of $\mathbf{a}^{T} \mathbf{s}(\omega)$ is 0 , the error metric $\xi$ can be expressed as a quadratic form in terms of a. In particular, we have

$$
\xi=\mathbf{a}^{T} \mathbf{P a}, \text { where } \mathbf{P}=\frac{1}{\pi} \int_{\mathcal{R}} W(\omega) \mathbf{s}(\omega) \mathbf{s}^{T}(\omega) d \omega
$$

and so the optimal denominator coefficient vector a can be found using Rayleigh's principle as before.

One problem with designing IIR filters not present when designing FIR filters is that of stability [49]. In order to have a causal, stable transfer function, all of its poles must lie inside the unit circle. In general, this constraint is very difficult to enforce. However, for all-pass filters, often times it is possible to obtain stable solutions by imposing constraints on the desired phase response $\phi_{H, \text { des }}(\omega)$. The phase of a real coefficient all-pass filter
$H(z)$ with $N_{\text {in }}$ poles inside the unit circle and $N_{\text {out }}$ poles outside the unit circle satisfies [22]

$$
\phi_{H}(\pi)=-\left(N_{\text {in }}-N_{\text {out }}\right) \pi .
$$

Hence, for a stable all-pass filter of order $N$, we have

$$
\phi_{H}(\pi)=-\int_{0}^{\pi} \tau_{H}(\omega) d \omega=-N \pi
$$

where $\tau_{H}(\omega)$ is the group delay of $H\left(e^{j \omega}\right)$. It was observed by Pei and Shyu [33], as well as by Nguyen et al. [22], that by constraining the desired group delay response $\tau_{H \text {,des }}(\omega)$ to satisfy (43), often times stable all-pass filters would be obtained. However, enforcing (43) for $\tau_{H \text {,des }}(\omega)$ does not guarantee stability.

Following an example considered by Pei and Shyu in [33], suppose that the desired phase response is

$$
\phi_{H, \mathrm{des}}(\omega)= \begin{cases}-85 \omega, & 0 \leq \omega<0.1 \pi \\ -65 \omega-2 \pi, & 0.1 \pi \leq \omega<0.7 \pi \\ -75 \omega+5 \pi, & 0.7 \pi \leq \omega<\pi\end{cases}
$$

It can be verified that $\phi_{H, \text { des }}(\omega)$ in (44) satisfies (43) for $N=$ 70. From this, it can be inferred that an all-pass filter of order 70 would be best suited to approximate $\phi_{H, \text { des }}(\omega)$ as in (44). In [33], the authors designed such an all-pass filter using the eigenfilter objective of (42), in which the weighing function $W(\omega)$ was chosen as

$$
W(\omega)= \begin{cases}90, & 0 \leq \omega \leq 0.08 \pi \\ 1, & 0.12 \pi \leq \omega \leq 0.685 \pi \\ 30, & 0.715 \pi \leq \omega \leq \pi \\ 0, & \text { otherwise }\end{cases}
$$

It should be noted that choosing $W(\omega)$ as in (45) introduces two transition bands into the design, namely $(0.08 \pi, 0.12 \pi)$ and $(0.685 \pi, 0.715 \pi)$, which were found necessary to yield a good phase approximation for the rest of the frequency region of interest. In Fig. 8(a), the group delay of the 70th-order all-pass eigenfilter designed using the above parameters is shown, whereas in Fig. 8(b), the phase error $\phi_{H \text {,des }}(\omega)-\phi_{H}(\omega)$ has been plotted. From both plots, we can see that the eigenfilter method yielded a good approximation to the desired phase. For the eigenfilter designed here, the pole farthest away from the origin had magnitude 0.9262 , and so the filter is stable. Despite the fact that in this example we have stability, it should be stressed that an all-pass filter designed using the eigenfilter method need not be stable, even if $\tau_{H \text {,des }}(\omega)$ satisfies (43).

2) Design of Arbitrary IIR Filters: Pei and Shyu [32], [41], [39], as well as Argenti and Del Re [1], [2] proposed different methods to design arbitrary IIR filters using the eigenfilter approach. In [41], Shyu and Pei proposed a method for designing multiband IIR filters using sums and differences of all-pass filters. The all-pass filter coefficients were found using a method similar to that described in the previous subsection. In [32], the authors proposed a time-domain method to approximate a desired impulse response $d(n)$, which need not be causal. How-

$$
\begin{aligned}
\mathbf{a} & \triangleq\left[\begin{array}{llll}
a_{0} & a_{1} & \cdots & a_{N}
\end{array}\right]^{T}, \\
\mathbf{s}(\omega) & \triangleq\left[\begin{array}{llll}
\sin \left(\phi_{A, \operatorname{des}}(\omega)\right) & \sin \left(\phi_{A, \operatorname{des}}(\omega)+\omega\right) & \cdots & \sin \left(\phi_{A, \operatorname{des}}(\omega)+N \omega\right)
\end{array}\right]^{T}
\end{aligned}
$$




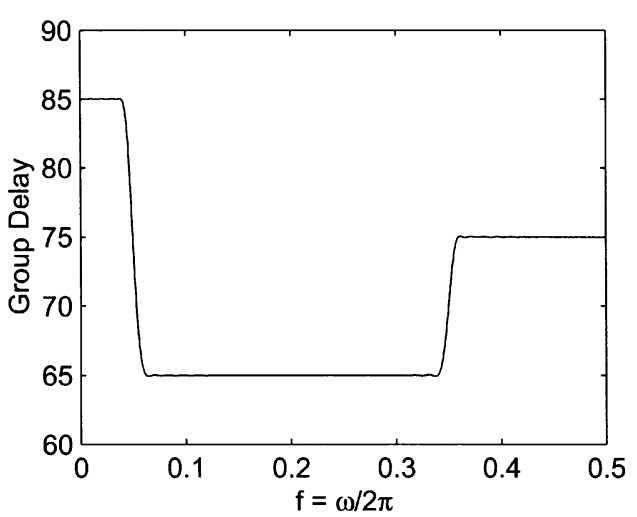

(a)

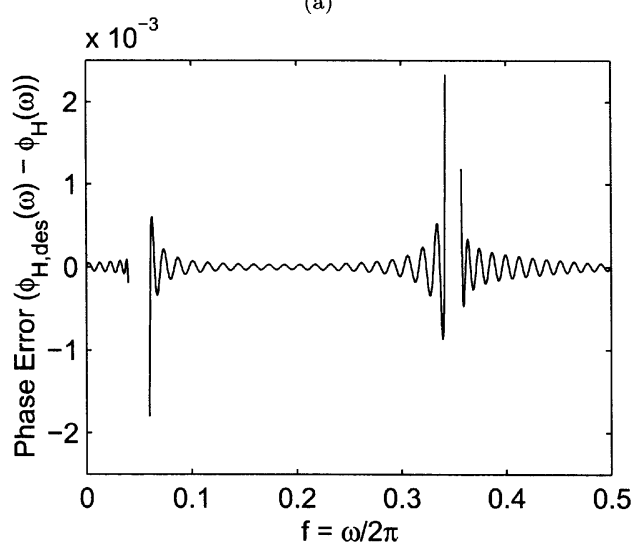

(b)

Fig. 8. (a) Group delay response of the all-pass eigenfilter. (b) Phase error $\phi_{H, \mathrm{des}}(\omega)-\phi_{H}(\omega)$. [N=70,W( $\left.\omega\right)$ as in (45)].

ever, to get good performance, computations must be performed on matrices of very large sizes (in [32], matrices of size 512 were used). Furthermore, there is no guarantee that the resulting filter will be stable. In [39], Pei and Shyu considered the design of special classes of IIR eigenfilters satisfying certain time and frequency-domain constraints. Recently, Argenti and Del Re [1], [2] proposed an IIR eigenfilter design method using a frequency-domain approach which we present here.

Suppose that we have a causal IIR filter $H(z)$ of the form

$$
\begin{aligned}
& H(z)=\frac{A(z)}{B(z)}, \\
& \text { where } A(z)=\sum_{n=0}^{M} a_{n} z^{-n} \text { and } B(z)=\sum_{n=0}^{N} b_{n} z^{-n} .
\end{aligned}
$$

Instead of choosing $A(z)$ and $B(z)$ to minimize the usual weighted mean-squared-error criterion of (26), which is difficult to do, the authors of [1] and [2] proposed choosing them to minimize the following error measure:

$$
\begin{aligned}
& \xi_{\mathrm{IIR}} \triangleq \frac{1}{2 \pi} \int_{\mathcal{R}} W(\omega)\left|\epsilon_{\mathrm{IIR}}(\omega)\right|^{2} d \omega, \\
& \text { where } \epsilon_{\mathrm{IIR}}(\omega) \triangleq D(\omega) B\left(e^{j \omega}\right)-A\left(e^{j \omega}\right) .
\end{aligned}
$$

Heuristically, $\xi_{\text {IIR }}$ can be argued to be a valid error criterion, since if $H\left(e^{j \omega}\right) \approx D(\omega)$, then we should also have $D(\omega) B\left(e^{j \omega}\right)-A\left(e^{j \omega}\right) \approx 0$ and conversely. The advantage here is that $\xi_{\text {IIR }}$ can be expressed as a quadratic form in terms of the polynomial coefficients, as we now show.
Define the following vectors:

$$
\begin{aligned}
\mathbf{a} & \triangleq\left[\begin{array}{llll}
a_{0} & a_{1} & \cdots & a_{M}
\end{array}\right]^{T}, \mathbf{b} \triangleq\left[\begin{array}{llll}
b_{0} & b_{1} & \cdots & b_{N}
\end{array}\right]^{T} \\
\mathbf{e}_{L}(z) & \triangleq\left[\begin{array}{llll}
1 & z^{-1} & \cdots & z^{-L}
\end{array}\right]^{T} .
\end{aligned}
$$

It should be noted that here we define the vector $(L+1) \times 1$ vector $\mathbf{e}_{L}(z)$ for any nonnegative integer $L$. Clearly, we have $A(z)=\mathbf{a}^{T} \mathbf{e}_{M}(z)$ and $B(z)=\mathbf{b}^{T} \mathbf{e}_{N}(z)$. As such, $\epsilon_{\mathrm{IIR}}(\omega)$ from (46) is simply

$$
\epsilon_{\mathrm{IIR}}(\omega)=D(\omega) \mathbf{b}^{T} \mathbf{e}_{N}\left(e^{j \omega}\right)-\mathbf{a}^{T} \mathbf{e}_{M}\left(e^{j \omega}\right) .
$$

Let us now define the following vector $\mathrm{c}$ obtained by concatenating $\mathbf{a}$ and $\mathbf{b}$ :

$$
\mathbf{c} \triangleq\left[\begin{array}{l}
\mathbf{a} \\
\mathbf{b}
\end{array}\right]
$$

Then we have

$$
\epsilon_{\mathrm{IIR}}(\omega)=\mathbf{c}^{T} \mathbf{d}(\omega), \text { where } \mathbf{d}(\omega) \triangleq\left[\begin{array}{c}
-\mathbf{e}_{M}\left(e^{j \omega}\right) \\
D(\omega) \mathbf{e}_{N}\left(e^{j \omega}\right)
\end{array}\right] .
$$

Using this in (46), we have

$$
\xi_{\text {IIR }}=\mathbf{c}^{\dagger} \mathbf{P c} \text {, where } \mathbf{P} \triangleq \frac{1}{2 \pi} \int_{\mathcal{R}} W(\omega) \mathbf{d}^{*}(\omega) \mathbf{d}^{T}(\omega) d \omega
$$

and hence $\xi_{\text {IIR }}$ is a quadratic form in terms of the vector of numerator and denominator coefficients. By subjecting $\mathbf{c}$ to the usual unit norm condition $\mathbf{c}^{\dagger} \mathbf{c}=1$, the optimal $\mathbf{c}$ is obtained using Rayleigh's principle.

As an example, suppose that the desired response is a low-pass filter with passband frequency $\omega_{p}$ and stopband frequency $\omega_{S}$ that has been delayed by $K$ for some $K \geq 0$. Namely, suppose that $D(\omega)$ is

$$
D(\omega)= \begin{cases}e^{-j K \omega}, & |\omega| \leq \omega_{p} \\ 0, & |\omega| \geq \omega_{S} \\ \text { don't care, } & \omega_{p}<|\omega|<\omega_{S} .\end{cases}
$$

Suppose that $\omega_{p}=0.3 \pi$ and $\omega_{S}=0.4 \pi$. If we choose $M=$ $12, N=5$, and $K=12$, the resulting magnitude, phase, and group delay plots of the IIR eigenfilter $H(z)$ are shown in Fig. 9(a)-(c), respectively. The phase and group delay are only plotted for the passband region, where we desire linear phase. From the plots, we see that the IIR eigenfilter has a relatively good magnitude response with a peak passband ripple size of about 0.08 and stopband attenuation of about $60 \mathrm{~dB}$, as well as very approximately linear phase in the passband region. The deviation of the phase from linearity is best seen from the group delay plot of Fig. 9(c). From this, we see that the phase becomes less linear as we approach the passband frequency $\omega_{p}$. Despite this, the fluctuations in the group delay in the passband are noticably less than those of elliptic and Chebyshev filters designed for the above observed passband and stopband ripple characteristics. To obtain this kind of performance with a linear-phase FIR filter, the filter order would have to be much larger than the ones used here, showing the merit of the designed IIR filter. However, it should be noted that the results are very sensitive to the choices of $M, N$, and $K$. Many choices yielded a poor response either in the magnitude or phase (or both) and many choices yielded an unstable filter. Here, the pole of $H(z)$ farthest away from the origin had magnitude 0.9334 and so $H(z)$ is stable. If we are not interested in the phase of $H(z)$ and we obtain an unstable filter with an adequate magnitude response, 


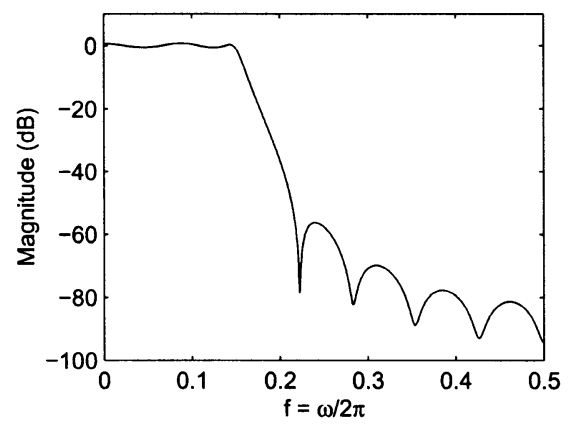

(a)

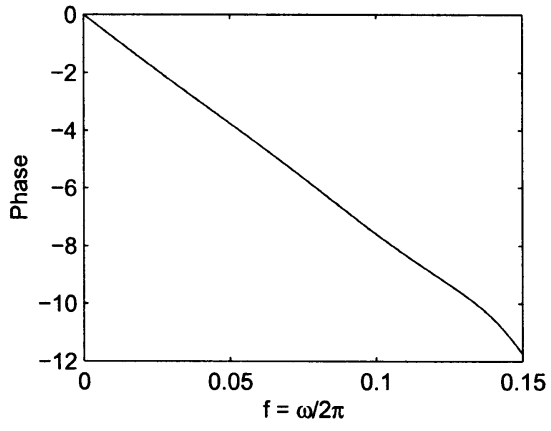

(b)

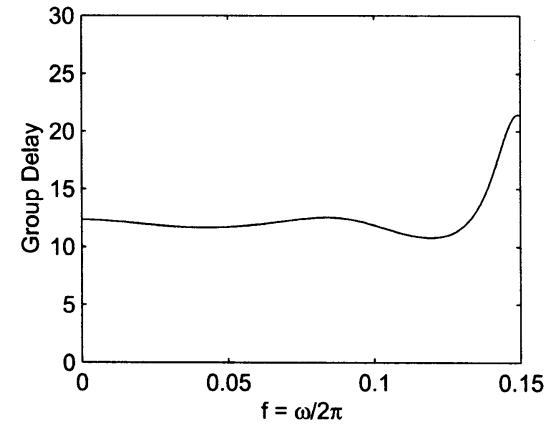

(c)

Fig. 9. (a) Magnitude response of the IIR low-pass eigenfilter. (b) Phase response in the passband region. (c) Group delay response in the passband region. $\left(\omega_{p}=0.3 \pi, \omega_{S}=0.4 \pi, M=12, N=5, K=12\right)$.

then we can replace each pole outside the unit circle with its reciprocal conjugate pair. In [1], [2], Argenti and Del Re proposed an iterative equiripple eigenfilter technique to improve the magnitude characteristic of the filter in this case, which was shown to work well through simulations. However, since we often desire exact or approximate linear phase in practice, IIR eigenfilters have not been as widely used as their FIR counterparts.

\section{E. Multidimensional Eigenfilters}

The eigenfilter method can easily be extended to the multidimensional case. Nashashibi and Charalambous [18] were the first to design multidimensional eigenfilters by considering the two-dimensional (2-D) case. Most, if not all, contributions regarding multidimensional eigenfilters have been for the 2-D case and have come from Pei and Shyu [26], [30], [31], [35], although others have also considered this problem, including $\mathrm{T}$. Chen [10], as well as H. Chen and Ford [8]. Here, we focus on the general $M$-dimensional case by generalizing the objective functions (22) and (27).

Suppose that we have an FIR $M$-dimensional signal $h(\mathbf{n})$, where $\mathbf{n}$ is an $M$-dimensional integer vector. By FIR, we mean that $h(\mathbf{n})$ is only nonzero for a finite number of integer vectors $\mathbf{n}$. The frequency response of $h(\mathbf{n})$ is a function $H(\omega)$ of $M$ variables given by the following [49]:

$$
H(\boldsymbol{\omega})=\sum_{\mathbf{n} \in \mathcal{N}_{0}} h(\mathbf{n}) e^{-j \boldsymbol{\omega}^{T} \mathbf{n}}
$$

where $\mathcal{N}_{0}$ is a subset of the set of all $M$-dimensional integer vectors. Here, $\mathcal{N}_{0}$ has finite cardinality and consists of those integer vectors $\mathbf{n}$ such that $h(\mathbf{n})$ is nonzero. If $H(\boldsymbol{\omega})$ has linear phase [49], then we have

$$
H(\boldsymbol{\omega})=c e^{-j \mathbf{k}^{T} \boldsymbol{\omega}} H_{R}(\boldsymbol{\omega})
$$

where $\mathbf{k}$ is a real constant vector and $H_{R}(\boldsymbol{\omega})$ is a real $M$-dimensional function of the form

$$
H_{R}(\boldsymbol{\omega})=\sum_{\mathbf{n} \in \mathcal{N}_{1}} b(\mathbf{n}) t(\boldsymbol{\omega}, \mathbf{n})
$$

where $\mathcal{N}_{1}$ is, like $\mathcal{N}_{0}$, a subset (of finite cardinality) of the set of all $M$-D integer vectors, $b(\mathbf{n})$ is a real sequence, and $t(\boldsymbol{\omega}, \mathbf{n})$ is a real function consisting of trigonometric functions (products of sines and cosines in particular). Note that if we impose some sort of lexicographical ordering on the coefficients of $h(\mathbf{n})$ for $H(\boldsymbol{\omega})$ as in (47) or $b(\mathbf{n})$ for $H_{R}(\boldsymbol{\omega})$ as in (48), then we have

$$
H(\boldsymbol{\omega})=\mathbf{h}^{T} \mathbf{e}(\boldsymbol{\omega}), H_{R}(\boldsymbol{\omega})=\mathbf{b}^{T} \mathbf{t}(\boldsymbol{\omega})
$$

where $\mathbf{h}, \mathbf{b}, \mathbf{e}(\boldsymbol{\omega})$, and $\mathbf{t}(\boldsymbol{\omega})$ consist of the respective elements of $h(\mathbf{n}), b(\mathbf{n}), e^{-j \boldsymbol{\omega}^{T} \mathbf{n}}$, and $t(\boldsymbol{\omega}, \mathbf{n})$ for appropriate $\mathbf{n}$ arranged according to some order.

If the goal is to design a filter to approximate a desired response $D(\boldsymbol{\omega})$, then one approach is to choose the filter coefficients to minimize either

$$
\xi_{r} \triangleq \frac{1}{\pi^{M}} \int_{\mathcal{R}} W(\boldsymbol{\omega})\left[\frac{D(\boldsymbol{\omega})}{D\left(\boldsymbol{\omega}_{0}\right)} H_{R}\left(\boldsymbol{\omega}_{0}\right)-H_{R}(\boldsymbol{\omega})\right]^{2} d \boldsymbol{\omega}
$$

if the desired response $D(\boldsymbol{\omega})$ is a real function, or alternatively

$$
\xi_{c} \triangleq \frac{1}{(2 \pi)^{M}} \int_{\mathcal{R}} W(\boldsymbol{\omega})\left|\frac{D(\boldsymbol{\omega})}{D\left(\boldsymbol{\omega}_{0}\right)} H\left(\boldsymbol{\omega}_{0}\right)-H(\boldsymbol{\omega})\right|^{2} d \boldsymbol{\omega}
$$

if $D(\boldsymbol{\omega})$ is complex. Note that $\xi_{r}$ and $\xi_{c}$ are simply $M$-dimensional extensions of the objectives (22) and (27) previously considered for the one-dimensional (1-D) case. Here, the region $\mathcal{R}$ in (50) is a subset of the $M$-dimensional interval $[0, \pi]^{M}$, whereas $\mathcal{R} \subseteq[0,2 \pi]^{M}$ in (51). Using the decompositions of $H_{R}(\boldsymbol{\omega})$ and $H(\boldsymbol{\omega})$ from (49), it can be shown that we get the equations shown at the bottom of the page. Hence, by imposing

$$
\begin{gathered}
\xi_{r}=\mathbf{b}^{T} \mathbf{P}_{r} \mathbf{b}, \text { where } \mathbf{P}_{r} \triangleq \frac{1}{\pi^{M}} \int_{\mathcal{R}} W(\boldsymbol{\omega})\left[\frac{D(\boldsymbol{\omega})}{D\left(\boldsymbol{\omega}_{0}\right)} \mathbf{t}\left(\boldsymbol{\omega}_{0}\right)-\mathbf{t}(\boldsymbol{\omega})\right]\left[\frac{D(\boldsymbol{\omega})}{D\left(\boldsymbol{\omega}_{0}\right)} \mathbf{t}\left(\boldsymbol{\omega}_{0}\right)-\mathbf{t}(\boldsymbol{\omega})\right]^{T} d \boldsymbol{\omega} \\
\xi_{c}=\mathbf{h}^{\dagger} \mathbf{P}_{c} \mathbf{h}, \text { where } \mathbf{P}_{c} \triangleq \frac{1}{(2 \pi)^{M}} \int_{\mathcal{R}} W(\boldsymbol{\omega})\left[\frac{D(\boldsymbol{\omega})}{D\left(\boldsymbol{\omega}_{0}\right)} \mathbf{e}\left(\boldsymbol{\omega}_{0}\right)-\mathbf{e}(\boldsymbol{\omega})\right]^{*}\left[\frac{D(\boldsymbol{\omega})}{D\left(\boldsymbol{\omega}_{0}\right)} \mathbf{e}\left(\boldsymbol{\omega}_{0}\right)-\mathbf{e}(\boldsymbol{\omega})\right]^{T} d \boldsymbol{\omega}
\end{gathered}
$$




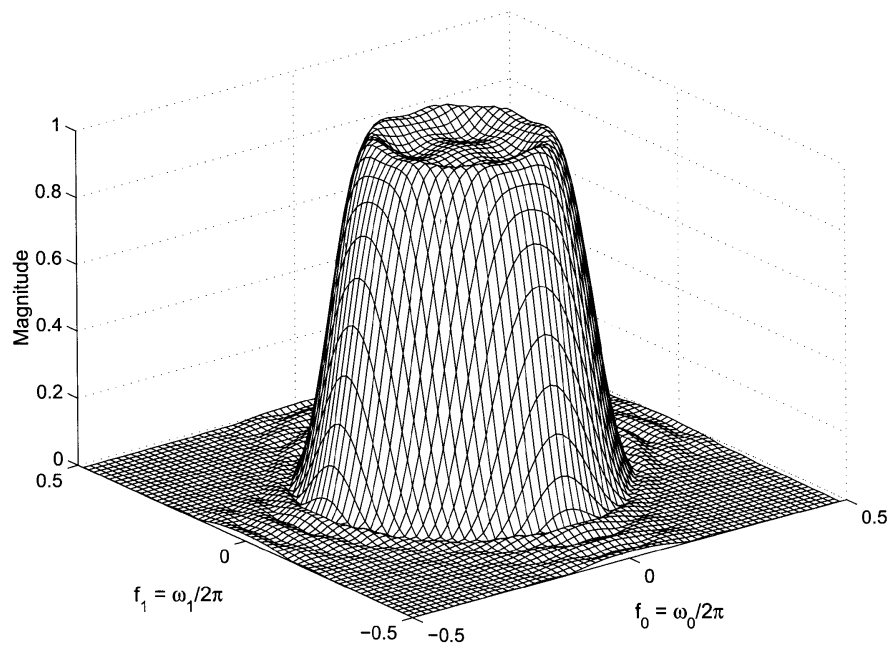

Fig. 10. Frequency response of a zero-phase $12 \times 12$ circular low-pass eigenfilter.

a unit norm constraint on $\mathbf{b}$ or $\mathbf{h}$, we can minimize $\xi_{r}$ or $\xi_{c}$ via Rayleigh's principle as before.

As an example, suppose that $D(\boldsymbol{\omega})$ is a 2-D circularly symmetric low-pass filter of the form

$$
D(\boldsymbol{\omega})= \begin{cases}1, & \omega_{0}^{2}+\omega_{1}^{2} \leq(0.4 \pi)^{2} \\ 0, & \omega_{0}^{2}+\omega_{1}^{2} \geq(0.6 \pi)^{2} \\ \text { don't care, } & \text { otherwise }\end{cases}
$$

The frequency response of a zero-phase $12 \times 12$ eigenfilter designed for this desired response is shown in Fig. 10. Here, equal uniform weighing was used and the reference frequency was chosen to be $\boldsymbol{\omega}_{0}=(0,0)$. As we can see, the eigenfilter method produced a good approximation to the desired response.

In addition to designing circularly symmetric filters, the eigenfilter method has been used to design a plethora of other multidimensional filters, most notably 2-D. Several of these include fan filters, elliptical filters, and quadrantally symmetric filters. An alternative method to design 2-D filters using a 1-D prototype with the McClellan transformation [15] has been considered by Pei and Shyu [31] as well as Chen and Ford [8].

\section{APPLICATIONS OF THE EIGENFILTER METHOD}

Thus far, we have only focused on the use of the eigenfilter method to design single filters of various desired responses. However, the eigenfilter approach has been found to be useful in other applications as well. For example, it has been used to design multistage IFIR [19] filters [9], arbitrary log magnitude and phase response filters [34], as well as prototype filters for uniform and nonuniform cosine-modulated filter banks [3], [4]. Eigenfilters have also been successfully employed for selective signal cancellation in acoustic environments [6], [7]. The eigenfilter technique has been shown useful for spectral/spatial selectivity, or beamforming, for antenna and microphone arrays [10], [11]. Recently, in image processing, eigenfilters which satisfy the Nyquist constraint have been shown to be suitable prototype filters for interpolation filters for image size conversion [53].

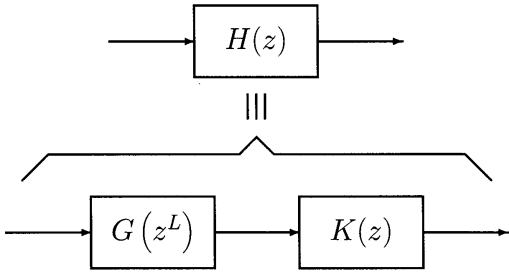

Fig. 11. IFIR filter implementation.

Among the various applications where the eigenfilter method has been shown to be beneficial, the one in which it has had the most impact is in communications. With the advent of DMT systems such as the digital subscriber loop (DSL) [43] in recent years, much attention has been given to the shortening of channels encountered in practice. For this problem, the eigenfilter method has been found to be very useful [17], [40], [5], [45]-[47]. Several such channel-shortening eigenfilters have been shown to be nearly optimal in terms of observed bit rate, which is the primary criterion of interest in DMT systems.

In this section, we will focus on three applications of the eigenfilter method. First, we will show how the eigenfilter approach can be applied to the design of multistage IFIR filters. Then, we show how the eigenfilter technique can be used for spectral/spacial beamforming. Finally, we will focus on applications in communications regarding the shortening of channels for DMT systems.

\section{A. Multistage IFIR Eigenfilters}

When designing linear-phase low-pass FIR filters, it can be shown, for fixed passband and stopband ripples sizes, that the filter order approximately varies inversely with the length of the transition band [49]. Namely, if $H(z)$ is a linear-phase low-pass filter of $\operatorname{order} N$, then $N \propto 1 / \Delta \omega_{H}$, where $\Delta \omega_{H} \triangleq \omega_{S}-\omega_{p}$. If the transition band length is relatively small, then designing a single filter to accomodate the given specifications often becomes cumbersome due to the large filter order required. Furthermore, as the filter order increases, design methods such as the McClellan-Parks algorithm and the least-squares approach become subject to numerical inaccuracies.

One simple way to overcome this problem is to implement the single FIR filter as a cascade of two filters as shown in Fig. 11, called the IFIR filter implementation [19]. Here, $L$ is a positive integer called the interpolation factor. The filter $G(z)$ is designed to be low pass with a passband frequency of $L \omega_{p}$ and a stopband frequency of $L \omega_{S}$ (assuming that $\omega_{S}<(\pi / L)$ ). As such, the expanded version $G\left(z^{L}\right)$ consists of a desired low-pass portion with passband and stopband frequencies $\omega_{p}$ and $\omega_{S}$, as well as $(L-1)$ undesired bandpass images [49] which occur by virtue of the expansion. These images are suppressed via the filter $K(z)$, which is designed to be low-pass with passband frequency $\omega_{p}$ and stopband frequency $(2 \pi / L)-\omega_{S}$.

The advantage to implementing $H(z)$ as $G\left(z^{L}\right) K(z)$ is that the transition bands of $G(z)$ and $K(z)$, denoted here by $\Delta \omega_{G}$ and $\Delta \omega_{K}$, may be much larger than $\Delta \omega_{H}$, thus transforming the problem of designing one filter with a high order into that of two filters with much smaller order. In particular, $\Delta \omega_{G}=$ 
$L\left(\omega_{S}-\omega_{p}\right)=L \Delta \omega_{H}$ and $\Delta \omega_{K}=(2 \pi / L)-\left(\omega_{p}+\omega_{S}\right)$, both of which are larger than $\Delta \omega_{H}$ assuming $\omega_{S}<(\pi / L)$.

In [9], Chen and Vaidyanathan showed how to design the filter $G(z)$ for the specifications of the original filter $H(z)$ using the eigenfilter method, assuming that the image suppressor $K(z)$ has already been designed. To properly describe their method, we must introduce a few new quantities. Suppose that $F(z)$ is some Type I linear-phase filter of order $Q$ for some even $Q$. Then

$$
\begin{aligned}
F\left(e^{j \omega}\right) & =\sum_{n=0}^{Q} f(n) e^{-j \omega n}=e^{-j \omega Q / 2} F_{R}(\omega), \\
\text { where } F_{R}(\omega) & =\sum_{n=0}^{Q / 2} s_{n} \cos \omega n .
\end{aligned}
$$

Using (8), we can express the $s_{n}$ 's in terms of the $f(n)$ 's. If $\mathbf{f} \triangleq$ $\left[\begin{array}{llll}f(0) & f(1) & \cdots & f(Q)\end{array}\right]^{T}$ is the vector of filter coefficients of $F(z)$ and $\mathbf{s} \triangleq\left[\begin{array}{cccc}s_{0} & s_{1} & \cdots & s_{Q / 2}\end{array}\right]^{T}$, then $\mathbf{s}$ is the folded version of $\mathbf{f}$ and we have

$$
\mathbf{f}=\mathbf{D}_{Q} \mathbf{s}, \mathbf{s}=\mathbf{E}_{Q} \mathbf{f}
$$

where $\mathbf{D}_{Q}$ and $\mathbf{E}_{Q}$ are, respectively, the $(Q+1) \times((Q / 2)+1)$ and $((Q / 2)+1) \times(Q+1)$ matrices

$$
\mathbf{D}_{Q} \triangleq \frac{1}{2}\left[\begin{array}{cc}
\mathbf{0} & \mathbf{J}_{Q / 2} \\
2 & \mathbf{0} \\
\mathbf{0} & \mathbf{I}_{Q / 2}
\end{array}\right], \mathbf{E}_{Q} \triangleq\left[\begin{array}{ccc}
\mathbf{0} & 1 & \mathbf{0} \\
\mathbf{J}_{Q / 2} & \mathbf{0} & \mathbf{I}_{Q / 2}
\end{array}\right]
$$

where $\mathbf{I}_{M}$ and $\mathbf{J}_{M}$ denote, respectively, the $M \times M$ identity and reversal matrices. With these definitions, we can now proceed to show how to design $G(z)$ for the specifications of $H(z)$ using the eigenfilter approach.

Suppose that $G(z)$ and $K(z)$ are real Type 1 linear-phase filters of orders $M$ and $J$. Then clearly $H(z)=G\left(z^{L}\right) K(z)$ is also a Type 1 linear-phase filter of order $N=L M+J$. Let $\mathbf{h}$ and $\mathrm{g}$ denote the vectors of coefficients of $H(z)$ and $G(z)$, and denote the folded versions of $\mathbf{h}$ and $\mathbf{g}$ by $\mathbf{b}$ and $\mathbf{c}$. Recall that the original goal is to make $H(z)$ a good low-pass narrowband filter. In [9], the authors considered choosing $H(z)$ using the eigenfilter approach of Section II. Namely, the folded version $\mathbf{b}$ (the same vector $\mathbf{b}$ considered in Section II) was chosen minimize the objective $\xi=\mathbf{b}^{T} \mathbf{P b}$ with $\mathbf{P}$ is as in (16), subject to the usual unit-norm condition $\mathbf{b}^{T} \mathbf{b}=1$. It was shown that this filter design problem could be posed as an eigenfilter problem in terms of the vector $\mathbf{c}$, the folded version of $\mathbf{g}$, as we now show.

First note that $\mathbf{g}=\mathbf{D}_{M} \mathbf{c}$ using (52) and (53). Then note that the coefficients of $G\left(z^{L}\right)$ are obtained by inserting $(L-1)$ consecutive zeros between each coefficient of $G(z)$. As such, if $\mathbf{g}^{\prime}$ denotes the vector of coefficients of $G^{\prime}(z) \triangleq G\left(z^{L}\right)$, then we have $\mathbf{g}^{\prime}=\mathbf{S}_{L} \mathbf{g}$, where $\mathbf{S}_{L}$ is the $(L M+1) \times(M+1)$ matrix

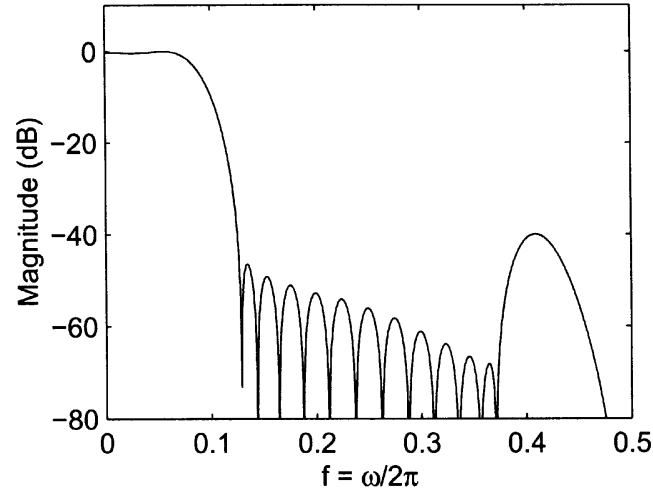

Fig. 12. Magnitude response of a low-pass IFIR eigenfilter.

consisting of the $(M+1)$ identity matrix with $(L-1)$ rows of zeros inserted between each row. For example

$$
\mathbf{S}_{2}=\left[\begin{array}{ccccc}
1 & 0 & 0 & \cdots & 0 \\
0 & 0 & 0 & \cdots & 0 \\
0 & 1 & 0 & \cdots & 0 \\
0 & 0 & 0 & \cdots & 0 \\
0 & 0 & 1 & \cdots & 0 \\
\vdots & \vdots & \vdots & \ddots & \vdots \\
0 & 0 & 0 & \cdots & 1
\end{array}\right] .
$$

Now note that the coefficients of $H(z)=G\left(z^{L}\right) K(z)=$ $K(z) G^{\prime}(z)$ can be expressed as $\mathbf{h}=\mathbf{K g}^{\prime}$, where $\mathbf{K}$ is the Toeplitz convolution matrix

$$
\mathbf{K}=\left[\begin{array}{cccc}
k_{0} & 0 & \cdots & 0 \\
k_{1} & k_{0} & \cdots & 0 \\
\vdots & \vdots & \ddots & \vdots \\
k_{J} & k_{J-1} & \ddots & k_{0} \\
0 & k_{J} & \ddots & \vdots \\
\vdots & \vdots & \ddots & k_{J-1} \\
0 & 0 & \cdots & k_{J}
\end{array}\right]
$$

consisting of the coefficients of the predesigned filter $K(z)=\sum_{n=0}^{J} k_{n} z^{-n}$. Hence, the folded version of $\mathbf{h}$, namely the vector $\mathbf{b}$, is given by the following:

$$
\mathbf{b}=\mathbf{E}_{N} \mathbf{h}=\mathbf{E}_{N} \mathbf{K g} \mathbf{g}^{\prime}=\mathbf{E}_{N} \mathbf{K S _ { L }} \mathbf{g}=\underbrace{\mathbf{E}_{N} \mathbf{K} \mathbf{S}_{L} \mathbf{D}_{M}}_{\mathbf{X}} \mathbf{c} .
$$

With this, the objective function $\xi$ from (16) becomes

$$
\xi=\mathbf{b}^{T} \mathbf{P b}=\mathbf{c}^{T} \underbrace{\mathbf{X}^{T} \mathbf{P X}}_{\mathbf{P}^{\prime}} \mathbf{c}=\mathbf{c}^{T} \mathbf{P}^{\prime} \mathbf{c} .
$$

To avoid trivial solutions, we subject $\mathbf{c}$ to the usual unit norm constraint $\mathbf{c}^{T} \mathbf{c}=1$. This shows that the eigenfilter problem for the original $H(z)$ can be posed as an eigenfilter problem for the model filter $G(z)$. We should note that once the optimal c is found, the resulting solution should be scaled so that $H\left(e^{j 0}\right)=1$.

As an example (taken from [9]), suppose we wish to design a low-pass filter with $\omega_{p}=0.15 \pi$ and $\omega_{S}=0.25 \pi$ using the IFIR eigenfilter approach. If we choose $\alpha=0.97, L=2, M=18$, and $J=6$ (so that $N=42$ ), we obtain the magnitude response shown in Fig. 12. Here, $K(z)$ was chosen to be a low-pass filter 
with equal and maximal flatness at $\omega=0, \pi[49]$. The resulting response has a passband ripple of $\delta_{p}=0.0641$ and a stopband ripple of $\delta_{S}=0.0105$ (or $-39.5449 \mathrm{~dB}$ ). To obtain the same specifications using a single eigenfilter would require an order of 36 [9]. One advantage from using the IFIR approach comes in the savings from implementing $H(z)$ in the cascade form $G\left(z^{L}\right) K(z)$. As $G(z)$ and $K(z)$ are linear phase of orders 18 and 6 , we only require $(18 / 2+1)+(6 / 2+1)=14$ multipliers and $18+6=24$ adders using the IFIR implementation [49]. In contrast, by using the single eigenfilter, we would require $(36 / 2+1)=19$ multipliers and 36 adders.

\section{B. Spectral/Spacial Beamforming}

A common problem in array signal processing is that of beamforming [13], in which a group of waves (such as electromagnetic or audio) impinge upon an array of sensors (such as antennas or microphones) and the goal is to tune the output of these sensors to focus only on a specific set of waves corresponding to a certain temporal frequency arriving from a certain spacial direction. For example, if a radio station is transmitting a signal in an environment with reflective paths between the transmitter and antenna array at the receiver, the receiver may wish to focus only on waves near the carrier frequency of the radio signal coming directly from the transmitter (following a path known as the line-of-sight path). It turns out that for delay-and-sum beamformers [13], such as the arc array with delay lines that we shall soon consider, the problem of steering the gain of the array to accept certain temporal frequencies and spacial directions while rejecting others is analogous to designing a filter to match a desired response. As a result, the eigenfilter approach can be applied to the problem of beamforming.

Chen was the first to consider using the eigenfilter method for the design of beamformers in [10]. In particular, he considered the design of an arc array with delay lines shown in Fig. 13. The array consists of $M$ sensors that are placed along a circle of radius $R$. As can be seen in Fig. 13, the $m$ th sensor is at an angle $\alpha_{m}$ with respect to a certain reference point and has $L_{m}$ delay lines attached to it. Each delay path is weighed by the quantity $w_{m, l}^{*}$ which is chosen at the receiver and used to steer the beam or gain of the array to a desired response. Waves from a particular source impinging upon the array are assumed to be in the far field and arrive at the array at an angle of $\theta$ as shown in Fig. 13. The signals from the various sensors and delay paths are combined to obtain a gain pattern $f(\theta, \omega)$ given by the following expression:

$$
f(\theta, \omega)=\sum_{m=0}^{M-1} \sum_{l=0}^{L_{m}-1} w_{m, l}^{*} e^{j \omega \tau_{m, l}(\theta)}
$$

where $\tau_{m, l}(\theta)$ is the delay of the $l$ th tap of the $m$ th sensor due to wave propagation and taps given by [10]

$$
\begin{aligned}
\tau_{m, l}(\theta)=-\frac{R}{c}\left[\cos \theta-\cos \left(\alpha_{m}-\theta\right)\right]-l T_{s}, & \\
0 & \leq m \leq M-1,0 \leq l \leq L_{m}-1 .
\end{aligned}
$$

Here, $c$ is the speed of the wave in the given medium (such as the speed of light or the speed of sound), $R$ is the radius

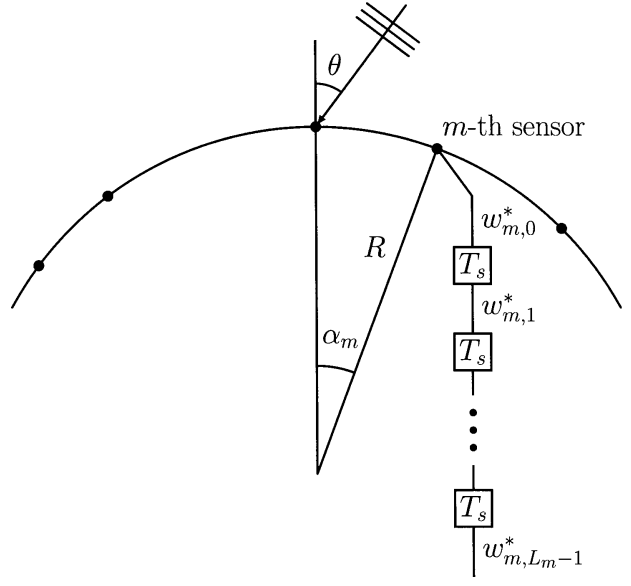

Fig. 13. Arc antenna array with delay lines.

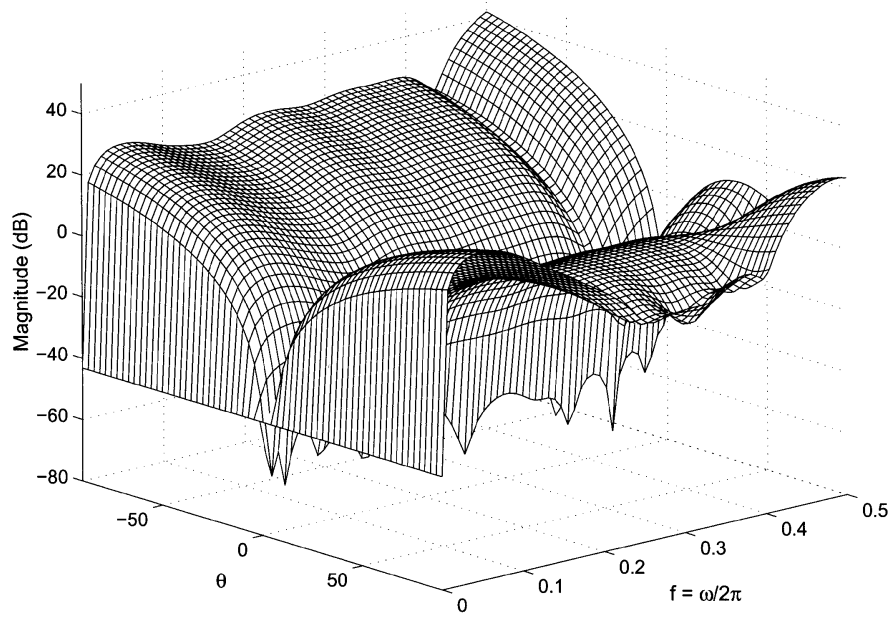

Fig. 14. Magnitude of the gain pattern of an arc array with delay lines.

of the arc array, and $T_{s}$ is the length of the unit delay. If we define the weight vector $\mathbf{w} \triangleq\left[\begin{array}{llll}w_{0} & w_{1} & \cdots & w_{N-1}\end{array}\right]^{T}$ where the $w_{i}$ 's are simply the $w_{m, l}$ 's arranged according to some order and similarly define the vector $\mathbf{b}(\theta, \omega) \triangleq\left[\begin{array}{llll}e^{j \omega \tau_{0}(\theta)} & e^{j \omega \tau_{1}(\theta)} & \cdots & e^{j \omega \tau_{N-1}(\theta)}\end{array}\right]^{T}$ where the $\tau_{i}(\theta)$ 's are just the $\tau_{m, l}(\theta)$ 's arranged according to the same order, then the gain pattern can be expressed as

$$
f(\mathbf{x})=\mathbf{w}^{\dagger} \mathbf{b}(\mathbf{x}), \text { where } \mathbf{x} \triangleq(\theta, \omega) .
$$

Analogous to the multidimensional objective of (51), Chen chose $\mathbf{w}$ to make $f(\mathbf{x})$ best approximate a desired gain $g(\mathbf{x})$ scaled by a factor $f\left(\mathbf{x}_{0}\right) / g\left(\mathbf{x}_{0}\right)$, where $\mathbf{x}_{0}$ is some reference point. Namely, $\mathbf{w}$ was chosen to minimize

$$
E \triangleq \int_{\mathbf{x} \in \mathcal{X}}\left|f(\mathbf{x})-f\left(\mathbf{x}_{0}\right) \frac{g(\mathbf{x})}{g\left(\mathbf{x}_{0}\right)}\right|^{2} d \mathbf{x} .
$$

As $f(\mathbf{x})=\mathbf{w}^{\dagger} \mathbf{b}(\mathbf{x})$, we clearly have

$E=\mathbf{w}^{\dagger} \mathbf{P} \mathbf{w}$, where $\mathbf{P}$

$=\int_{\mathbf{x} \in \mathcal{X}}\left[\mathbf{b}(\mathbf{x})-\mathbf{b}\left(\mathbf{x}_{0}\right) \frac{g(\mathbf{x})}{g\left(\mathbf{x}_{0}\right)}\right]\left[\mathbf{b}(\mathbf{x})-\mathbf{b}\left(\mathbf{x}_{0}\right) \frac{g(\mathbf{x})}{g\left(\mathbf{x}_{0}\right)}\right]^{\dagger} d \mathbf{x}$. 


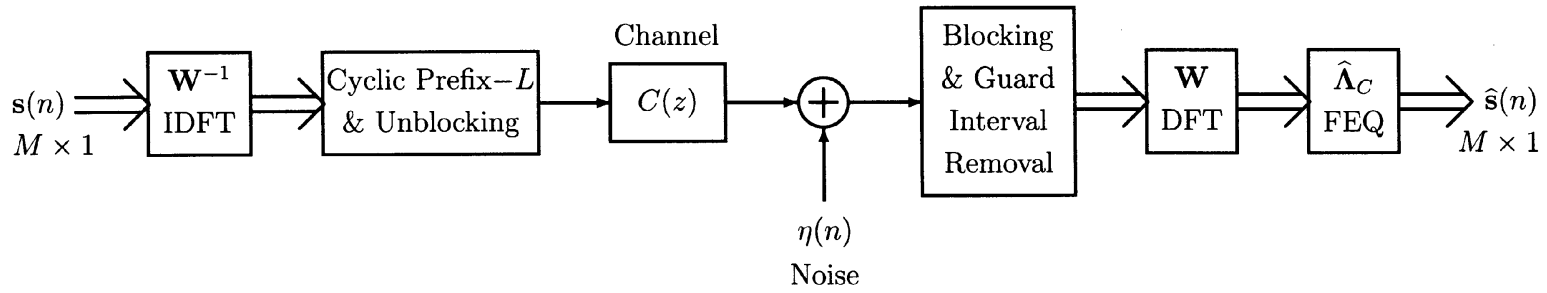

$$
\begin{gathered}
\widehat{\boldsymbol{\Lambda}}_{C} \text { is diagonal with } \\
{\left[\widehat{\boldsymbol{\Lambda}}_{C}\right]_{k, k}=\frac{1}{C\left(e^{j \omega_{k}}\right)}, \omega_{k}=\frac{2 \pi k}{M}, 0 \leq k \leq M-1}
\end{gathered}
$$

Fig. 15. Typical DMT system.

With the usual unit norm constraint on $\mathbf{w}$, the optimal $\mathbf{w}$ can be found using Rayleigh's principle as before.

As an example (adapted from [10]), suppose that we have an arc array with ten sensors $(M=10)$, each attached with ten delay taps $\left(L_{m}=10\right.$ for all $m$ ). Let the $\alpha_{m}$ be chosen to be $0^{\circ}$, $2^{\circ}, 4^{\circ}, 6^{\circ}, 8^{\circ}, 10^{\circ},-2^{\circ},-5^{\circ},-8^{\circ}$, and $-11^{\circ}$. Suppose that we desire the response to be unity in the range $41^{\circ} \leq \theta \leq 49^{\circ}$ and $0.4 \pi \leq \omega \leq 0.8 \pi$, whereas we desire zero gain for $-3^{\circ} \leq \theta \leq$ $3^{\circ}$ for all $\omega$. In other words, $g(\mathbf{x})$ is given by

$$
g(\mathbf{x})= \begin{cases}1, & 41^{\circ} \leq \theta \leq 49^{\circ}, 0.4 \pi \leq \omega \leq 0.8 \pi \\ 0 & -3^{\circ} \leq \theta \leq 3^{\circ}, \forall \omega \\ \text { don't care, } & \text { otherwise. }\end{cases}
$$

For the fictitious example in which $R=0.1, c=1$, and $T_{s}=1$, the optimal gain pattern we obtain is shown in Fig. 14. As we can see, though the gain pattern takes on a wide range of values in the "don't care" regions, the gain in the regions of interest match closely with the desired response.

\section{Channel-Shortening Eigenfilter Equalizers for DMT Systems}

A typical DMT system is shown in Fig. 15 [43]. Recently this communications system has received much attention on account of its low complexity and excellent performance compared to traditional modem systems. Practical DMT systems such as the asynchronous digital subscriber line (ADSL), have become very popular and have revolutionized telephone wireline communications.

Perhaps the most important feature of the DMT system of Fig. 15 is the inclusion of redundancy in the form of a cyclic prefix of length $L$ [43]. The beauty of this redundancy is that it can be shown that in the absence of noise, we have perfect reconstruction, i.e., $\widehat{\mathbf{s}}(n)=\mathbf{s}(n)$, if the channel $C(z)$ is of length less than or equal to $L+1$. Essentially, the DMT system is able to equalize an FIR channel using only FIR components (namely the blocking components along with the DFT matrices $\mathbf{W}$ and $\mathbf{W}^{-1}$ as well as the frequency-domain equalizer). This is only possible because of the inherent redundancy.

From a practical point of view, we want the cyclic prefix length $L$ as small as possible, since it represents a redundancy which hinders the overall rate of the system by a factor $M /(M+L)$. However, the channel may be very long, as is the case in practical DMT systems such as ADSL in which the

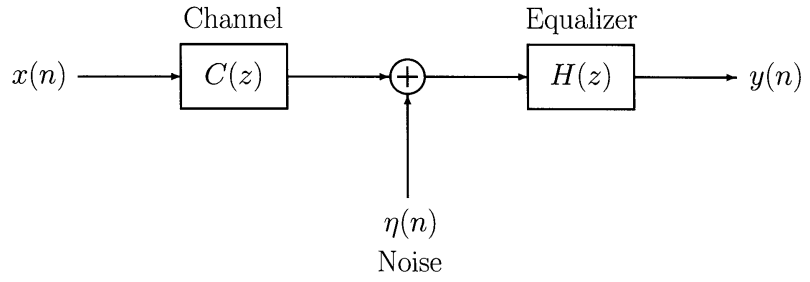

Fig. 16. Channel/equalizer model.

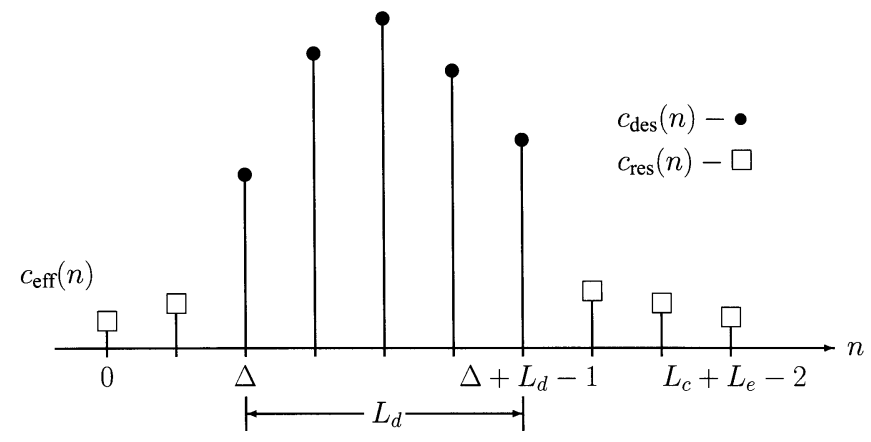

Fig. 17. Decomposition of the effective channel $c_{\text {eff }}(n)$ into a desired channel $c_{\text {des }}(n)$ and residual channel $c_{\text {res }}(n)$.

channel is a telephone wire line [43]. For example, in ADSL, $M=512$ and $L=32$ [43], although the channels are typically hundreds of samples long. This suggests the need for an equalizer at the receiver which shortens the channel, as shown in Fig. 16. Such an equalizer is typically called a time-domain equalizer or TEQ [43]. As the channel may have zeros near or outside the unit circle, the equalizer $H(z)$ is usually not chosen to exactly shorten the channel $C(z)$, as this may result in spurious noise amplification. Instead, $H(z)$ is typically an FIR filter chosen to concentrate the energy of the effective channel $C_{\text {eff }}(z) \triangleq H(z) C(z)$ in a window of length $L+1$.

1) The Channel-Shortening Problem: Suppose that the length of the channel $c(n)$ is $L_{c}$ and that of the equalizer $h(n)$ is $L_{e}$. Then, the effective channel $c_{\mathrm{eff}}(n)=h(n) * c(n)$ is of length $L_{\text {eff }} \triangleq\left(L_{c}+L_{e}-1\right)$. The goal is to shorten the channel to a length $L_{d}<L_{c}$, i.e., to concentrate most of the energy of $c_{\text {eff }}(n)$ to a window of length $L_{d}$. We can decompose $c_{\text {eff }}(n)$ as a sum of two responses, namely a desired response $c_{\text {des }}(n)$ which is exactly of length $L_{d}$, and a residual response $c_{\text {res }}(n)$ which consists of whatever remains of $c_{\mathrm{eff}}(n)$ after removing the desired response $c_{\mathrm{des}}(n)$. This is illustrated in Fig. 17. Here, $\Delta$ is a delay parameter which satisfies $0 \leq \Delta \leq L_{\text {eff }}-L_{d}$. 
Melsa et al. [17], chose the equalizer coefficients to minimize the energy of the residual response $c_{\text {res }}(n)$ subject to keeping the energy of the desired response $c_{\text {des }}(n)$ at unity. As we now show, this problem can be posed as an eigenfilter problem. Define the following vectors and matrices:

$$
\begin{aligned}
\mathbf{h} & \triangleq\left[\begin{array}{llll}
h(0) & h(1) & \cdots & h\left(L_{e}-1\right)
\end{array}\right]^{T} \\
\mathbf{c}_{\mathrm{eff}} & =\left[\begin{array}{llll}
c_{\mathrm{eff}}(0) & c_{\mathrm{eff}}(1) & \cdots & c_{\mathrm{eff}}\left(L_{\mathrm{eff}}-1\right)
\end{array}\right]^{T} \\
\mathbf{C} \triangleq & {\left[\begin{array}{cccc}
c(0) & 0 & \cdots & 0 \\
c(1) & c(0) & \cdots & 0 \\
\vdots & \vdots & \ddots & \vdots \\
c\left(L_{c}-1\right) & c\left(L_{c}-2\right) & \ddots & c(0) \\
0 & c\left(L_{c}-1\right) & \ddots & \vdots \\
\vdots & \vdots & \ddots & c\left(L_{c}-2\right) \\
0 & 0 & \cdots & c\left(L_{c}-1\right)
\end{array}\right] . }
\end{aligned}
$$

Here, $\mathbf{h}, \mathbf{c}_{\mathrm{eff}}$, and $\mathbf{C}$ are, respectively, $L_{e} \times 1, L_{\mathrm{eff}} \times 1$, and $L_{\text {eff }} \times L_{e}$. By the convolution $c_{\mathrm{eff}}(n)=h(n) * c(n)$

$$
\mathbf{c}_{\text {eff }}=\text { Ch. }
$$

Now define the following vectors $\mathbf{c}_{\text {des }}$ and $\mathbf{c}_{\text {res }}$, as well as the windowing matrices $\mathbf{W}_{\Delta}$ and $\overline{\mathbf{W}}_{\Delta}$, as shown in the equation at the bottom of the page. Here, $\mathbf{c}_{\mathrm{des}}$ and $\mathbf{c}_{\mathrm{res}}$ are $L_{\mathrm{eff}} \times 1$, whereas $\mathbf{W}_{\Delta}$ and $\overline{\mathbf{W}}_{\Delta}$ are $L_{\text {eff }} \times L_{\text {eff }}$. Clearly, we have $\mathbf{c}_{\text {des }}=$ $\mathbf{W}_{\Delta} \mathbf{c}_{\text {eff }}=\mathbf{W}_{\Delta} \mathbf{C h}$ and $\mathbf{c}_{\text {res }}=\overline{\mathbf{W}}_{\Delta} \mathbf{c}_{\text {eff }}=\overline{\mathbf{W}}_{\Delta} \mathbf{C h}$, where we have used the convolution equation of (54). The energy of the desired and residual responses, which we denote here, respectively, by $E_{\mathrm{des}}$ and $E_{\mathrm{res}}$, are simply the following:

$$
\begin{gathered}
E_{\mathrm{des}} \triangleq \sum_{n}\left|c_{\mathrm{des}}(n)\right|^{2}=\mathbf{c}_{\mathrm{des}}^{\dagger} \mathbf{c}_{\mathrm{des}}=\mathbf{h}^{\dagger} \underbrace{\left(\mathbf{C}^{\dagger} \mathbf{W}_{\Delta} \mathbf{C}\right)}_{\mathbf{B}} \mathbf{h} \\
E_{\mathrm{res}} \triangleq \sum_{n}\left|c_{\mathrm{res}}(n)\right|^{2}=\mathbf{c}_{\mathrm{res}}^{\dagger} \mathbf{c}_{\mathrm{res}}=\mathbf{h}^{\dagger} \underbrace{\left(\mathbf{C}^{\dagger} \overline{\mathbf{W}}_{\Delta} \mathbf{C}\right)}_{\mathbf{A}} \mathbf{h} .
\end{gathered}
$$

Here we have used the fact that $\mathbf{W}_{\Delta}^{\dagger} \mathbf{W}_{\Delta}=\mathbf{W}_{\Delta}$ and $\overline{\mathbf{W}}_{\Delta}^{\dagger} \overline{\mathbf{W}}_{\Delta}=\overline{\mathbf{W}}_{\Delta}$. Hence, the design problem considered by Melsa et al. [17] is tantamount to the following problem:

$$
\text { Minimize } E_{\text {res }}=\mathbf{h}^{\dagger} \mathbf{A h} \text {, subject to } \mathbf{h}^{\dagger} \mathbf{B h}=1
$$

where $\mathbf{A}$ and $\mathbf{B}$ are Hermitian positive semidefinite matrices. To show that the problem of (55) can be posed as a traditional eigenfilter problem, we perform a Cholesky decomposition of the matrix $\mathbf{B}$ [12]. Assuming that the matrix $\mathbf{B}$ is positive definite, then it admits a Cholesky-like decomposition of the form [12]

$$
\mathbf{B}=\mathbf{T}^{\dagger} \mathbf{T}
$$

where $\mathbf{T}$ is a square nonsingular matrix. If we define the vector $\mathbf{v} \triangleq \mathbf{T h}$, so that $\mathbf{h}=\mathbf{T}^{-1} \mathbf{v}$, then as $\mathbf{h}$ varies over all nonzero vectors, $\mathbf{v}$ will as well. Hence, the problem in (55) is equivalent to

$$
\begin{aligned}
& \text { Minimize } E_{\text {res }}=\mathbf{v}^{\dagger} \mathbf{P} \mathbf{v} \text {, subject to } \mathbf{v}^{\dagger} \mathbf{v}=1, \\
& \text { where } \mathbf{P} \triangleq\left(\mathbf{T}^{-1}\right)^{\dagger} \mathbf{A}\left(\mathbf{T}^{-1}\right) .
\end{aligned}
$$

This problem (in terms of the vector $\mathbf{v}$ ) is in fact the eigenfilter problem, which can be solved via Rayleigh's principle as before. Once the optimal $\mathbf{v}$ is found, the optimal $\mathbf{h}$ is found using $\mathbf{h}=\mathbf{T}^{-1} \mathbf{v}$. If the matrix $\mathbf{B}$ is strictly positive semidefinite, the original optimization problem of (55) can still be posed as an eigenfilter problem [17]; however, the method by which this is done is much more complicated.

The method for channel shortening given in (55) is known as the maximum shortening signal-to-noise ratio (MSSNR) method [5], since it maximizes the shortening signal-to-noise ratio defined by

$$
\mathrm{SSNR} \triangleq \frac{E_{\mathrm{des}}}{E_{\mathrm{res}}} .
$$

In Fig. 18, the impulse response of a typical channel encountered in a DMT system is shown, along with the equalized effective channel designed using the MSSNR method. Here, the channel is carrier-service area (CSA) loop \# 1, a common subscriber loop encountered in an ADSL system [43]. The channel is of length $L_{c}=512$, the equalizer is of length 16 , and the desired length is $L_{d}=33$ (corresponding to a cyclic prefix length of 32). Here, the optimal equalizer was calculated for all $\Delta$ in the range $0 \leq \Delta \leq 40$ and the actual $\Delta$ used was the one which yielded the largest SSNR. As can be seen, the effective channel appears to be "shortened" to the desired length. In this example, the best equalizer occured when $\Delta$ was 25 corresponding to a SSNR of $33.3312 \mathrm{~dB}$.

$$
\begin{aligned}
& \mathbf{c}_{\mathrm{des}} \triangleq\left[\begin{array}{llll}
c_{\mathrm{des}}(0) & c_{\mathrm{des}}(1) & \cdots & c_{\mathrm{des}}\left(L_{\mathrm{eff}}-1\right)
\end{array}\right]^{T} \\
& =\left[\begin{array}{llllllllll}
0 & \cdots & 0 & c_{\mathrm{eff}}(\Delta) & c_{\mathrm{eff}}(\Delta+1) & \cdots & c_{\mathrm{eff}}\left(\Delta+L_{d}-1\right) & 0 & \cdots & 0
\end{array}\right]^{T} \\
& \mathbf{c}_{\mathrm{res}} \triangleq\left[\begin{array}{llll}
c_{\mathrm{res}}(0) & c_{\mathrm{res}}(1) & \cdots & c_{\mathrm{res}}\left(L_{\mathrm{eff}}-1\right)
\end{array}\right]^{T} \\
& =\left[\begin{array}{lllllllllll}
c_{\mathrm{eff}}(0) & c_{\mathrm{eff}}(1) & \cdots & c_{\mathrm{eff}}(\Delta-1) & 0 & \cdots & 0 & c_{\mathrm{eff}}\left(\Delta+L_{d}\right) & c_{\mathrm{eff}}\left(\Delta+L_{d}+1\right) & \cdots & c_{\mathrm{eff}}\left(L_{\mathrm{eff}}-1\right)
\end{array}\right]^{T} \\
& \mathbf{W}_{\Delta} \triangleq\left[\begin{array}{ccc}
\mathbf{0}_{\Delta} & \mathbf{0} & \mathbf{0} \\
\mathbf{0} & \mathbf{I}_{L_{d}} & \mathbf{0} \\
\mathbf{0} & \mathbf{0} & \mathbf{0}_{L_{\mathrm{eff}}-L_{d}-\Delta}
\end{array}\right] \\
& \overline{\mathbf{W}}_{\Delta} \triangleq \mathbf{I}_{L_{\text {eff }}}-\mathbf{W}_{\Delta}
\end{aligned}
$$




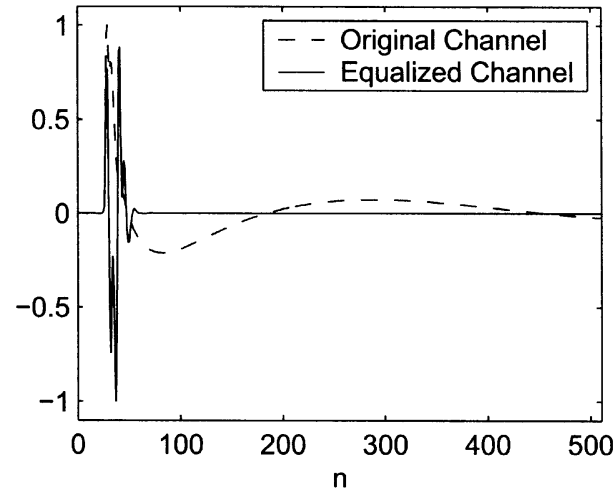

Fig. 18. Impulse responses of the original channel $c(n)$ and the equalized channel $c_{\text {eff }}(n)$. $\left(L_{c}=512, L_{e}=16, L_{d}=33, \Delta=25\right.$, SSNR $=$ $33.3312 \mathrm{~dB})$.

Several generalizations of the MSSNR method for channel shortening have been proposed recently. In [47], the authors proposed a method to jointly shorten the channel and suppress the noise power observed after equalization, whereas in [45], a similar method was proposed requiring only one Cholesky decomposition (i.e., the decomposition did not depend on the delay parameter $\Delta$ ). Recently, a novel eigenfilter method called the minimum intersymbol interference (min-ISI) method was proposed by Arslan et al. [5], which exploited the DMT subchannels which cannot be used to improve the DMT channels used for joint channel shortening and noise suppression. Simulation results have shown that these methods perform nearly optimally in terms of observed bit rate (the primary figure of merit for DMT systems), especially the min-ISI method.

\section{CONCLUding REMARKS}

The eigenfilter method has been shown to possess several advantages over other traditional filter design methods. As opposed to the least-squares approach, which requires the computation of a matrix inverse which may be susceptible to numerical inaccuracies, the eigenfilter method has a much lower design complexity and remains robust even when ill-conditioned matrices are present in the design problem. In contrast to the McClellan-Parks algorithm, which is difficult to modify for certain design criteria, the eigenfilter method can easily be modified to satisfy a plethora of design constraints. Such advantages, when coupled together with the good performance of eigenfilter method, make it an attractive method to use for filter design.

In addition to its numerous strengths, the eigenfilter method was shown to be useful in a variety of applications, since many design problems can be posed as an eigenfilter problem. Here, the method was shown to be useful for designing model filters for multistage IFIR filters, which are useful when narrow transition bands are required. Also, the eigenfilter method was shown to be applicable to the problem of spectral/spacial filtering or beamforming for sensor arrays, in which we wish to focus on temporal frequencies in a particular band arriving from a particular spacial direction. Finally, the method was found to be useful for the design of channel-shortening equalizers, which are commonly needed in popular communications systems such as ADSL.

The eigenfilter method continues to find applications in several areas of research. Recently, the eigenfilter method for channel shortening was extended for the design of fractionally spaced equalizers [45], [47] as well as for designing channel-shortening equalizers for multiple-input multiple-output (MIMO) channels [46]. In addition, the eigenfilter method has been shown to appear in the design of certain optimum compaction filters [51]. Current open problems in filter bank theory include using the eigenfilter method to design FIR compaction filters, using IFIR eigenfilters as compaction filters, and using the eigenfilter method to design optimal filter banks. As can be seen, on account of its numerous merits, together with the myriad of design problems to which it can be applied, the eigenfilter method is and will continue to be a versatile design algorithm.

\section{SELECTED REFERENCES BY TOPIC}

Early Works on Eigenfilters:

[42] D. Slepian, 1978

[50] P. P. Vaidyanathan and T. Q. Nguyen, 1987

[18] A. Nashashibi and C. Charalambous, 1988

[24] S. C. Pei and J. J. Shyu, 1988

Arbitrary Desired Response Eigenfilters:

[20] T. Q. Nguyen, 1991

[29] S. C. Pei and J. J. Shyu, 1993

[21] T. Q. Nguyen, 1993

\section{All-Pass Eigenfilters:}

[14] T. I. Laakso, T. Q. Nguyen, and R. D. Koilpillai, 1993

[33] S. C. Pei and J. J. Shyu, 1994

[22] T. Q. Nguyen, T. I. Laakso, and R. D. Koilpillai, 1994

[55] X. Zhang and H. Iwakura, 1999

\section{IIR Eigenfilters:}

[41] J. J. Shyu and S. C. Pei, 1992

[32] S. C. Pei and J. J. Shyu, 1994

[1] F. Argenti and E. Del Re, 1998

[2] F. Argenti and E. Del Re, 1998

[39] S. C. Pei, C. C. Hsu, and P. H. Wang, 2002

\section{Multidimensional Eigenfilters:}

[18] A. Nashashibi and C. Charalambous, 1988

[26] S. C. Pei and J. J. Shyu, 1990

[30] S. C. Pei and J. J. Shyu, 1993

[10] T. Chen, 1993

[31] S. C. Pei and J. J. Shyu, 1993

[35] S. C. Pei and J. J. Shyu, 1994

[8] H. Chen and G. E. Ford, 1996

Applications in Spectral/spacial Filtering or Beamforming: [10] T. Chen, 1993

[6] S. Bharitkar and C. Kyriakakis, 2000

[7] S. Bharitkar and C. Kyriakakis, 2001

[11] S. Doclo and M. Moonen, 2002

\section{Applications in Channel-Shortening Equalizer Design:}

[17] P. J. W. Melsa, R. C. Younce, and C. E. Rohrs, 1996

[40] R. Schur and J. Speidel, 2001

[5] G. Arslan, B. L. Evans, and S. Kiaei, 2001

[45] A. Tkacenko and P. P. Vaidyanathan, 2002

[46] A. Tkacenko and P. P. Vaidyanathan, 2002

[47] A. Tkacenko and P. P. Vaidyanathan, 2002 


\section{REFERENCES}

[1] F. Argenti and E. D. Re, "Design of IIR eigenfilters with arbitrary magnitude frequency response," Proc. IEEE ICASSP '98, vol. 3, pp. $1265-1268,1998$.

[2] —-, "Design of IIR eigenfilters in the frequency domain," IEEE Trans. Signal Processing, vol. 46, pp. 1694-1698, June 1998.

[3] _ - "Eigenfilter design of real and complex coefficient prototypes for uniform and nonuniform filter banks," Proc. IEEE ICASSP '99, vol. 3, pp. 1477-1480, 1999.

[4] - "Eigenfilter design of real and complex coefficient QMF prototypes," IEEE Trans. Circuits Syst. II, vol. 47, pp. 787-792, Aug. 2000.

[5] G. Arslan, B. L. Evans, and S. Kiaei, "Equalization for discrete multitone transceivers to maximize bit rate," IEEE Trans. Signal Processing, vol. 49, pp. 3123-3135, Dec. 2001

[6] S. Bharitkar and C. Kyriakakis, "Eigenfilters for signal cancellation," Proc. IEEE ISPACS 'O0, 2000.

[7] — "Correlation and spatial sensitivity of eigenfilters for selective signal cancellation in multiple-listener environments," in Proc. Asilomar Conf. Signals, Systems, and Computers, vol. 2, Monterey, CA, Nov. 2001, pp. 1578-1582.

[8] H. Chen and G. E. Ford, "A unified eigenfilter approach to the design of two-dimensional zero-phase FIR filters with the Mcclellan transform," IEEE Trans. Circuits Syst. II, vol. 43, pp. 622-626, Aug. 1996.

[9] T. Chen and P. P. Vaidyanathan, "Design of IFIR eigenfilters," Proc. IEEE ISCAS '91, vol. 1, pp. 264-267, 1991.

[10] T. Chen, "Unified eigenfilter approach: With applications to spectral/spatial filtering," Proc. IEEE ISCAS '93, vol. 1, pp. 331-334, 1993.

[11] S. Doclo and M. Moonen, "Comparison of least-squares and eigenfilter techniques for broadband beamforming," Proc. 3rd IEEE SPS-2002, pp. 73-76, 2002.

[12] R. A. Horn and C. R. Johnson, Matrix Analysis. Cambridge, U.K: Cambridge Univ. Press, 1985.

[13] D. H. Johnson and D. E. Dudgeon, Array Signal Processing. Englewood Cliffs, NJ: Prentice-Hall, Inc., 1993

[14] T. I. Laakso, T. Q. Nguyen, and R. D. Koilpillai, "Designing allpass filters using the eigenfilter method," Proc. IEEE ICASSP '93, vol. 3, pp. 77-80, 1993 .

[15] J. H. McClellan, "The design of 2-D digital filters by transformations," in Proc. 7th Annu. Princeton Conf. Information Science and Systems, 1973, pp. 247-251.

[16] J. H. McClellan and T. Parks, "A unified approach to the design of optimum FIR linear-phase digital filters," IEEE Trans. Circuit Theory, vol. CT-20, pp. 697-701, Nov. 1973.

[17] P. J. W. Melsa, R. C. Younce, and C. E. Rohrs, "Impulse response shortening for discrete multitone transceivers," IEEE Trans. Commun., vol. 44, pp. 1662-1672, Dec. 1996.

[18] A. Nashashibi and C. Charalambous, "2-D FIR eigenfilters," Proc. IEEE ISCAS '88, vol. 2, pp. 1037-1040, 1988.

[19] Y. Neuvo, C. Y. Dong, and S. K. Mitra, "Interpolated finite impulse response filters," IEEE Trans. Acoust., Speech, Signal Processing, pp. 563-570, June 1984.

[20] T. Q. Nguyen, "The eigenfilter for the design of linear-phase filters with arbitrary magnitude response," Proc. IEEE ICASSP '91, vol. 3, pp. 1981-1984, 1991.

[21] $\ldots$, "The design of arbitrary FIR digital filters using the eigenfilter method," IEEE Trans. Signal Processing, vol. 41, pp. 1128-1139, Mar. 1993.

[22] T. Q. Nguyen, T. I. Laakso, and R. D. Koilpillai, "Eigenfilter approach for the design of allpass filters approximating a given phase response," IEEE Trans. Signal Processing, vol. 42, pp. 2257-2263, Sept. 1994.

[23] M. Okuda, M. Ikehara, and S. Takahashi, "Fast and stable least-squares approach for the design of linear phase FIR filters," IEEE Trans. Signal Processing, vol. 46, pp. 1485-1493, June 1998.

[24] S. C. Pei and J. J. Shyu, "Design of FIR Hilbert transformers and differentiators by eigenfilter," IEEE Trans. Circuits Syst., vol. 35, pp. $1457-1461$, Nov. 1988.

[25] — , "Eigenfilter design of higher-order digital differentiators," IEEE Trans. Acoust., Speech, Signal Processing, vol. 37, no. 4, pp. 505-511, Apr. 1989.

[26] - "2-D FIR eigenfilters: A least-squares approach," IEEE Trans. Circuits Syst., vol. 37, pp. 24-34, Jan. 1990.

[27] — - "Eigen-approach for designing FIR filters and all-pass phase equalizers with prescribed magnitude and phase response," IEEE Trans. Circuits Syst. II, vol. 39, pp. 137-145, Mar. 1992.
[28] _- "Analytic closed forms for designing higher order digital differentiators by eigen-approach," Proc. IEEE ISCAS '92, vol. 2, pp. 573-576, 1992

[29] _ "Complex eigenfilter design of arbitrary complex coefficient FIR digital filters," IEEE Trans. Circuits Syst. II, vol. 40, pp. 32-40, Jan. 1993.

[30] — - "Design of two-dimensional FIR eigenfilters for sampling-structure conversion," IEEE Trans. Circuits Syst. Video Technol., vol. 3, pp. 158-162, Apr. 1993.

[31] _ - "Design of 2-D FIR digital filters by Mcclellan transformation and least squares eigencontour mapping," IEEE Trans. Circuits Syst. II, vol. 40, pp. 546-555, Sept. 1993.

[32] _ "Design of 1-D and 2-D IIR eigenfilters," IEEE Trans. Signal Processing, vol. 42, pp. 962-966, Apr. 1994.

[33] — , "Eigenfilter design of 1-D and 2-D IIR digital all-pass filters," IEEE Trans. Signal Processing, vol. 42, pp. 966-968, Apr. 1994.

[34] — , "Design of arbitrary FIR log eigenfilters," IEEE Trans. Circuits Syst. II, vol. 41, pp. 361-364, May 1994.

[35] — "A unified approach to the design of quadrantally symmetric linear-phase two-dimensional FIR digital filters by eigenfilter approach," IEEE Trans. Signal Processing, vol. 42, pp. 2886-2890, Oct. 1994.

[36] _ - "Analytic closed-form matrix for designing higher order digital differentiators using eigen-approach," IEEE Trans. Signal Processing, vol. 44, no. 3, pp. 698-701, Mar. 1996.

[37] S. C. Pei, C. C. Tseng, and W. S. Yang, "FIR filter design with linear constraints using the eigenfilter approach," IEEE Trans. Circuits Syst. II, vol. 45, pp. 232-237, Feb. 1998.

[38] S. C. Pei and C. C. Tseng, "A new eigenfilter based on total least squares error criterion," IEEE Trans. Circuits Syst. I, vol. 48, pp. 699-709, June 2001.

[39] S. C. Pei, C. C. Hsu, and P. H. Wang, "Design of a class of IIR eigenfilters with time- and frequency-domain constraints," IEEE Trans. Circuits Syste. II, vol. 49, pp. 145-151, Feb. 2002.

[40] R. Schur and J. Speidel, "An efficient equalization method to minimize delay spread in OFDM/DMT systems," Proc. IEEE ICC '01, vol. 1, pp. $1-5$, Jun. 2001

[41] J. J. Shyu and S. C. Pei, "Design of IIR multi-band filters using IIR all-pass eigenfilters," in Proc. 35th Midwest Symp. Circuits and Systems, vol. 1, Aug. 1992, pp. 601-604.

[42] D. Slepian, "Prolate spheroidal wave functions, Fourier analysis, and uncertainty - V: The discrete case," Bell Sys. Tech. J., vol. 57, pp. 1371-1430, May-June 1978.

[43] T. Starr, J. M. Cioffi, and P. J. Silverman, Understanding Digital Subscriber Line Technology. Englewood Cliffs, NJ: Prentice-Hall, 1999.

[44] C. W. Therrien, Discrete Random Signals and Statistical Signal Processing. Englewood Cliffs, NJ: Prentice-Hall, 1992.

[45] A. Tkacenko and P. P. Vaidyanathan, "Noise optimized eigenfilter design of time-domain equalizers for DMT systems," Proc. IEEE ICC '02, Apr. 2002.

[46] - "Eigenfilter design of mimo equalizers for channel shortening," Proc. IEEE ICASSP' '02, May 2002.

[47] — "A new eigenfilter based method for optimal design of channel shortening equalizers," Proc. IEEE ISCAS '02, May 2002, submitted for publication.

[48] D. W. Tufts and J. T. Francis, "Designing digital lowpass filters: Comparison of some methods and criteria," IEEE Trans. Audio Electroacoust., vol. AU-18, pp. 487-494, Dec. 1970.

[49] P. P. Vaidyanathan, Multirate Systems and Filter Banks. Englewood Cliffs, NJ: Prentice-Hall, 1993.

[50] P. P. Vaidyanathan and T. Q. Nguyen, "Eigenfilters: A new approach to least-squares FIR filter design and applications including Nyquist filters," IEEE Trans. Circuits Syst., vol. 34, pp. 11-23, Jan. 1987.

[51] P. P. Vaidyanathan, "Theory of optimal orthonormal subband coders," IEEE Trans. Signal Processing, vol. 46, pp. 1528-1543, June 1998.

[52] Y. Wisutmethangoon and T. Q. Nguyen, "A method for design of $M$ th-band filters," IEEE Trans. Signal Processing, vol. 47, pp. 1669-1678, June 1999.

[53] S. Yang and T. Q. Nguyen, "Interpolated $M$ th-band filters for image size conversion," IEEE Trans. Signal Processing, vol. 50, pp. 3028-3035, Dec. 2002.

[54] C. Zhang and T. Chen, "Toward optimal least square filters using the eigenfilter approach," Proc. IEEE ICASSP '02, vol. 4, pp. 4171-4174, 2002 .

[55] X. Zhang and H. Iwakura, "Design of IIR digital allpass filters based on eigenvalue problem," IEEE Trans. Signal Processing, vol. 47, pp. 554-559, Feb. 1999. 


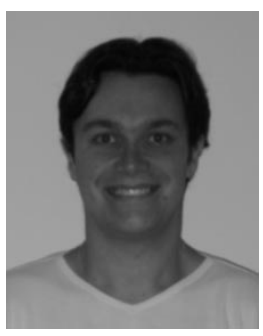

Andre Tkacenko (S'00) was born in Santa Clara, CA, on February 24, 1977. He received the B.S. and M.S. degrees in electrical engineering from the California Institute of Technology (Caltech), Pasadena, in 1999 and 2001, respectively. He is currently working toward the Ph.D. degree in the field of digital signal processing at Caltech.

In 1999, he was awarded the Graduate Division Fellowship from Caltech. His research interests include multirate systems, wavelet transforms, and their applications in digital communications and

data compression

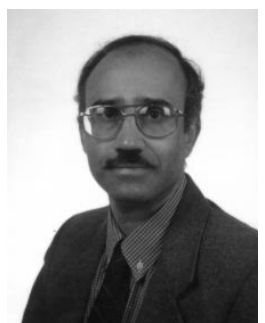

P. P. Vaidyanathan (S'80-M'83-SM'88-F'91) was born in Calcutta, India, on Oct. 16, 1954. He received the B.Sc. (Hons.) degree in physics and the B.Tech and M.Tech. degrees in radiophysics and electronics, all from the University of Calcutta, Calcutta, India, in 1974, 1977, and 1979, respectively, and the Ph.D degree in electrical and computer engineering from the University of California at Santa Barbara in 1982.

He was a post-doctoral fellow at the University of California, Santa Barbara, from September 1982 to March 1983. In March 1983 he joined the Electrical Engineering Department, Calfornia Institute of Technology, Pasadena, as an Assistant Professor, and since 1993 has been Professor of Electrical Engineering there. He is the author of the book Multirate Systems and Filter Banks (Englewood Cliffs, NJ: Prentice Hall, 1993). He has written several chapters for various signal processing handbooks and has authored a number of papers in IEEE journals. His main research interests are in digital signal processing, multirate systems, wavelet transforms, and signal processing for digital communications.

Dr. Vaidyanathan was an Associate Editor for the IEEE TRANSACTIONS ON CIRCUITS AND Systems during 1985-1987, and is currently an Associate Editor for IEEE Signal Processing Letters, and a Consulting Editor for Applied and Computational Harmonic Analysis. In 1998, he was a Guest Editor for special issues of the IEEE TRANSACTIONS ON SIGNAL PROCESSING and the IEEE TRANSACTIONS ON CiRCUITS AND SySTEMS-II: ANALOG AND DigiTAL SignAL PROCESSING, on the topics of filter banks, wavelets, and subband coders. He served as Vice Chairman of the Technical Program Committee for the 1983 IEEE International symposium on Circuits and Systems, and as the Technical Program Chairman for the 1992 IEEE International Symposium on Circuits and Systems. He was a recepient of the Award for Excellence in Teaching at the California Institute of Technology in 1983-1984, 1992-1993, and 1993-1994. He also received the National Science Foundation's Presidential Young Investigator Award in 1986, the IEEE Acoustics, Speech, and Signal Processing Senior Award for his paper on multirate perfect-reconstruction filter banks in 1989, and the S. K. Mitra Memorial Award from the Institute of Electronics and Telecommuncations Engineers, India, for his joint paper in the IETE Journal in 1990. He was also the coauthor of a paper on linear-phase perfect reconstruction filter banks in the IEEE SP Transactions, for which the first author (Truong Nguyen) received the Young outstanding author award in 1993. Dr. Vaidyanathan was elected Fellow of the IEEE in 1991. He received the 1995 F. E. Terman Award of the American Society for Engineering Education, sponsored by Hewlett Packard Company, for his contributions to engineering education, especially the book Multirate systems and filter banks published by Prentice Hall in 1993. He has given several plenary talks including at the Sampta'01, Eusipco'98, SPCOM'95, and Asilomar' 88 conferences on signal processing. He has been chosen a distinguished lecturer for the IEEE Signal Processing Society for the year 1996-97. In 1999 he was chosen to receive the IEEE CAS Society's Golden Jubilee Medal. $\mathrm{He}$ is a recepient of the IEEE Signal Processing Society's Technical Achievement Award for the year 2002
Truong Q. Nguyen (S'85-M'85-SM'95) received the B.S., M.S., and Ph.D degrees in electrical engineering from the California Institute of Technology, Pasadena, in 1985, 1986 and 1989, respectively.

He was with MIT Lincoln Laboratory, Lexington, MA, from June 1989 to July 1994, as a Member of Technical Staff. During the academic year 1993-1994, he was a Visiting Lecturer at MIT and an Adjunct Professor at Northeastern University, Boston, MA. From August 1994 to July 1998, he was with the Electrical and Computer Engineering Department, University of Wisconsin, Madison. He was with Boston University, Boston, MA, from August 1996 to June 2001. He is currently a Professor at the Electrical and Computer Engineering Department, University of California at San Diego. He is coauthor (with Prof. G. Strang) of the textbook Wavelets and Filter Banks (Wellesley, MA: Wellesley-Cambridge Press, 1997) and the author of several matlab-based toolboxes on image compression, electrocardiogram compression, and filter bank design. He has published over 100 publications. He is currently the Series Editor (Digital Signal Processing) for Academic Press. His research interests include video processing algorithms and their efficient implementation.

Dr. Nguyen served as an Associate Editor for the IEEE TRANSACTIONS ON Signal PROCESSING from 1994-1996 and for the IEEE TRANSACTIONS ON CIRCUITS AND SYSTEMS from 1996-1997. He is currently an Associate Editor for the IEEE Transactions on IMAge Processing, IEEE TRANSACtions ON CIRCUITS AND SYSTEMS-II: ANALOG AND DigITAL SignAl PROCESSING, and IEEE Signal Processing Letters. He received the IEEE TRANSACTIONS ON Signal PROCESSING Paper Award (in the image and multidimensional processing area) for the paper he co-wrote with Prof. Vaidyanathan on linear-phase perfect-reconstruction filter banks in 1992 and the National Science Foundation Career Award in 1995. 